\title{
Anti-biofilm Properties of Bacterial Di-Rhamnolipids and Their Semi-Synthetic Amide Derivatives
}

\section{OPEN ACCESS}

Edited by:

Noton Kumar Dutta,

Johns Hopkins University,

United States

Reviewed by:

Amit Kumar Mandal,

Raiganj University, India

Esther Orozco

Centro de Investigación y de Estudios

Avanzados del IPN, Mexico

Dinesh Sriramulu,

Shres Consultancy (Life Sciences),

India

*Correspondence:

Lidija Senerovic

seneroviclidija@imgge.bg.ac.rs

Jasmina Nikodinovic-Runic

jasmina.nikodinovic@imgge.bg.ac.rs

Specialty section:

This article was submitted to Antimicrobials, Resistance

and Chemotherapy,

a section of the journal

Frontiers in Microbiology

Received: 05 September 2017 Accepted: 27 November 2017

Published: 08 December 2017

Citation:

Aleksic I, Petkovic M, Jovanovic M,

Milivojevic $D$, Vasiljevic $B$,

Nikodinovic-Runic J and Senerovic L

(2017) Anti-biofilm Properties

of Bacterial Di-Rhamnolipids

and Their Semi-Synthetic Amide

Derivatives. Front. Microbiol. 8:2454.

doi: 10.3389/fmicb.2017.02454
Ivana Aleksic ${ }^{1}$, Milos Petkovic ${ }^{2}$, Milos Jovanovic ${ }^{2}$, Dusan Milivojevic ${ }^{1}$, Branka Vasiljevic ${ }^{1}$, Jasmina Nikodinovic-Runic ${ }^{1 *}$ and Lidija Senerovic ${ }^{1 *}$

${ }^{1}$ Institute of Molecular Genetics and Genetic Engineering, University of Belgrade, Belgrade, Serbia, ${ }^{2}$ Department of Organic Chemistry, Faculty of Pharmacy, University of Belgrade, Belgrade, Serbia

A new strain, namely Lysinibacillus sp. BV152.1 was isolated from the rhizosphere of ground ivy (Glechoma hederacea L.) producing metabolites with potent ability to inhibit biofilm formation of an important human pathogens Pseudomonas aeruginosa PAO1, Staphylococcus aureus, and Serratia marcescens. Structural characterization revealed di-rhamnolipids mixture containing rhamnose (Rha)-Rha-C10-C10, Rha-Rha-C8-C10, and Rha-Rha-C10-C12 in the ratio 7:2:1 as the active principle. Purified di-rhamnolipids, as well as commercially available di-rhamnolipids (Rha-Rha-C10-C10, 93\%) were used as the substrate for the chemical derivatization for the first time, yielding three semisynthetic amide derivatives, benzyl-, piperidine-, and morpholine. A comparative study of the anti-biofilm, antibacterial and cytotoxic properties revealed that di-Rha from Lysinibacillus sp. BV152.1 were more potent in biofilm inhibition, both cell adhesion and biofilm maturation, than commercial di-rhamnolipids inhibiting $50 \%$ of $P$. aeruginosa PAO1 biofilm formation at $50 \mu \mathrm{g} \mathrm{mL}^{-1}$ and $75 \mu \mathrm{g} \mathrm{mL}^{-1}$, respectively. None of the dirhamnolipids exhibited antimicrobial properties at concentrations of up to $500 \mu \mathrm{g} \mathrm{mL}^{-1}$. Amide derivatization improved inhibition of biofilm formation and dispersion activities of di-rhamnolipids from both sources, with morpholine derivative being the most active causing more than $80 \%$ biofilm inhibition at concentrations $100 \mu \mathrm{g} \mathrm{mL}{ }^{-1}$. Semisynthetic amide derivatives showed increased antibacterial activity against $S$. aureus, and also showed higher cytotoxicity. Therefore, described di-rhamnolipids are potent anti-biofilm agents and the described approach can be seen as viable approach in reaching new rhamnolipid based derivatives with tailored biological properties.

Keywords: rhamnolipids, di-rhamnolipids, biofilms, cell adhesion, amide derivative

\section{INTRODUCTION}

Microbial biofilms are prevalent in nature, especially in medical, industrial and environmental settings where they cause undesirable effects due to their pathogenicity and resistance toward antibiotics or biofouling technologies (Berlanga and Guerrero, 2016; Holscher and Kovacs, 2017). Biofilms play an important role in virulence of many pathogenic bacteria including Pseudomonas aeruginosa, Staphylococcus aureus, and Serratia marcescens (Costerton et al., 1999; Singh et al., 2000; Bryers, 2008). They can be formed in the patients' tissues or on the surface of medical 
devices associated with the human body such as catheters, nasolaryngeal tubes, or stents (Bryers, 2008). Various strategies have been developed and employed for the efficient biofilm control (Coughlan et al., 2016; Rowson and Townsend, 2016), however, new, natural, and effective anti-biofilm agents are still highly sought after. Application of microbial biosurfactants in antibiofilm and antibiofouling efforts emerged as an attractive and one of the viable strategies in recent years (Vatsa et al., 2010; Varjani and Upasani, 2017).

Biotechnological interest in biosurfactants has grown exponentially since the 1980s when these molecules were found useful in oil recovery and bioremediation (Abdel-Mawgoud et al., 2010; Muller et al., 2012; Dobler et al., 2016). Rhamnolipids as a group of anionic microbial glycolipids, consisting of L-(+)-rhamnose and $\beta$-hydroxyalkanoic acids, have been considered in a wide range of applications such as health care, cosmetics, pharmaceutical processes, food and beverage processing, detergents and polymer industry, cryo-protectants, biofuels, microbial fuel cells, and bioremediation, due to their unique characteristics such as low toxicity, surface activities, sustainability, and biodegradability (Li, 2017; Varjani and Upasani, 2017).

Rhamnolipids were firstly described as secondary metabolites of $P$. aeruginosa in 1949 and were found essential for the growth of this bacterium on hydrophobic carbon sources (Muller et al., 2011; Gong et al., 2015), but are also critical for forming structured biofilms with pore and channels in this organism (Davey et al., 2003). So far, over 60 rhamnolipid congeners and homologs have been described, including mono- and di-rhamnolipids, containing mostly two, one, or even three molecules of $\beta$-hydroxyalkanoic acids with varying carbon chain length (Abdel-Mawgoud et al., 2010). Rhamnolipids reached the market as an active ingredient of Zonix TM fungicide (Jeneil Biosurfactants Co., Saukville, WI, United States), and there is a number of reports regarding their antibacterial and anti-biofilm activities (Abdel-Mawgoud et al., 2010; Vatsa et al., 2010; Kim et al., 2015; Zhong et al., 2015). Dirhamnolipids (di-Rha), as a class of rhamnolipids, are less studied but received attention as they showed therapeutic properties for wound healing and ulcer treatment (Stipcevic et al., 2006; Piljac et al., 2008). Stable rhamnolipid production has been achieved with $P$. aeruginosa and certain Burkholderia strains (Chrzanowski et al., 2012; Dobler et al., 2016), however, the identification and the development of other rhamnolipid producing bacteria is an important issue to avoid potential pathogenicity and to broaden the spectrum of the products.

In this study, we sought to identify secondary metabolites amongst metabolites of new bacterial isolates that would have efficient anti-biofilm activity preferably without the effect on bacterial growth, to avoid the development of resistance. As the most activity has been associated with the di-rhamnolipids, the investigation has been extended to the semi-synthetic preparation of di-rhamnolipids amide derivatives and comprehensive assessment of their anti-biofilm, antibacterial, and cytotoxic activities.

\section{MATERIALS AND METHODS}

\section{Materials}

Chemicals and reagents including rhamnolipids mixture R90 (AGAE Technologies) and 4-(Dimethylamino)pyridine (DMAP) were purchased from Sigma-Aldrich (Munich, Germany). Reagents used for coupling reactions including $N$ (3-Dimethylaminopropyl)- $N^{\prime}$-ethylcarbodiimide hydrochloride (EDCI) were purchased from ACROS Organics (Morris Plains, NJ, United States). Unless otherwise stated, all media components were purchased either from Oxoid (Cambridge, United Kingdom) or Becton Dickinson (Sparks, MD, United States).

Gentamycin sulfate and chloramphenicol were purchased from Sigma-Aldrich (Munich, Germany), tetracycline hydrochloride from USB Co. (Cleveland, OH, United States) and cycloheximide from SERVA (Heidelberg, Germany). The stock solutions of antibiotics were prepared as follows: gentamycin $30 \mathrm{~g}$ $\mathrm{L}^{-1}$ in water, tetracycline $15 \mathrm{~g} \mathrm{~L}^{-1}$ in ethanol, chloramphenicol $50 \mathrm{~g} \mathrm{~L}^{-1}$ in ethanol, and cycloheximide $70 \mathrm{~g} \mathrm{~L}^{-1}$ in water and were kept at $-20^{\circ} \mathrm{C}$.

\section{Isolation and Identification of Bacterium BV152.1}

Bacteria were isolated from the rhizosphere of medicinal plant Glechoma hederacea L. (ground ivy), using previously described procedure (Djokic et al., 2011). The medium for isolation of bacteria was starch casein agar containing soluble starch (10 $\left.\mathrm{g} \mathrm{L}^{-1}\right)$, casein $\left(1 \mathrm{~g} \mathrm{~L}^{-1}\right), \mathrm{KH}_{2} \mathrm{PO}_{4}\left(0.5 \mathrm{~g} \mathrm{~L}^{-1}\right), \mathrm{MgSO}_{4}$ $\left(0.5 \mathrm{~g} \mathrm{~L}^{-1}\right), \mathrm{NaCl}\left(3 \mathrm{~g} \mathrm{~L}^{-1}\right)$, and bacteriological agar $\left(15 \mathrm{~g} \mathrm{~L}^{-1}\right)$, supplemented with antifungal cycloheximide $\left(50 \mu \mathrm{g} \mathrm{L}^{-1}\right)$. Plates were incubated at $30^{\circ} \mathrm{C}$ for 7 days.

Isolates were grown in JS broth containing soy flour (30 $\left.\mathrm{g} \mathrm{L}^{-1}\right)$, soluble starch $\left(20 \mathrm{~g} \mathrm{~L}^{-1}\right)$, glucose $\left(20 \mathrm{~g} \mathrm{~L}^{-1}\right)$, mannitol $\left(15 \mathrm{~g} \mathrm{~L}^{-1}\right)$, and $\mathrm{CaCO}_{3}\left(10 \mathrm{~g} \mathrm{~L}^{-1}\right)$ for 7 days, at $30^{\circ} \mathrm{C}$ on a rotary shaker $(180 \mathrm{rpm})$ and their cultures were extracted with equal volume of ethyl acetate and screened for the inhibition of biofilm formation of $P$. aeruginosa PAO1.

Genomic DNA isolation, PCR amplification of 16 S rRNA gene sequencing and sequence alignment were performed as described previously (Stankovic et al., 2012).

\section{Preparation and Analysis of BV152.1 Culture Extracts}

Spore suspensions of BV152.1 were prepared in glycerol [20\%, v/v, (Kieser et al., 2000)] $(20 \mu \mathrm{l})$ and firstly inoculated into vegetative medium (tryptone soy broth, $8 \mathrm{~g} \mathrm{~L}^{-1}$, yeast extract, $4 \mathrm{~g} \mathrm{~L}^{-1}$, maltose, $15 \mathrm{~g} \mathrm{~L}^{-1}$, and $\mathrm{CaCO}_{3}, 2 \mathrm{~g} \mathrm{~L}^{-1}$ ) and incubated at $30^{\circ} \mathrm{C}$ for $48 \mathrm{~h}$, and this pre-culture was then used for the inoculation of JS broth $(1 \%, \mathrm{v} / \mathrm{v})$. Cultures were grown in Erlenmeyer flasks (1:5 culture to volume ratio) containing coiled stainless steel spring for better aeration and incubated at $30^{\circ} \mathrm{C}$ on a rotary shaker (180 rpm) for 7 days.

Culture $(1 \mathrm{~L})$ was extracted using the equal volume of ethyl acetate by vigorous shaking for $30 \mathrm{~min}$. The organic phase was then separated, dried under vacuum and further purified by flash 
chromatography. Flash chromatography employed silica gel 60 (230-400 mesh) and the following solvent system: n-hexane and ethyl acetate (3:1 ratio, $100 \mathrm{ml}), \mathrm{n}$-hexane and ethyl acetate (1:1 ratio, $100 \mathrm{ml})$, ethyl acetate and methanol (9:1 ratio, $100 \mathrm{ml})$, followed by pure methanol $(100 \mathrm{ml})$. Collected fractions were analyzed by thin layer chromatography, which was carried out using alumina plates with $0.25 \mathrm{~mm}$ silica layer (Kieselgel 60 $\mathrm{F}_{254}$, Merck, Darmstadt, Germany) and the appropriate fractions were combined, dried under vacuum, and weighted. Anti-biofilm activity was determined for each fraction.

\section{Purification and Characterization of Di-Rhamnolipids}

The fraction that showed anti-biofilm forming activity was purified further using the following solvents: pure ethyl acetate (50 mL); ethyl acetate, dichloromethane, and methanol (8:1:1 ratio, $50 \mathrm{~mL}$ ) and ethyl acetate, dichloromethane, and methanol (4:2:1 ratio, $50 \mathrm{~mL})$. The most active fraction (F3) was further analyzed by a combination of NMR and mass spectrometry. The NMR spectra were recorded on a Bruker Ascend $400(400 \mathrm{MHz})$ spectrometer. Chemical shifts are given in parts per million $(\delta)$ downfield from tetramethylsilane as the internal standard and deuterochloroform as a solvent.

Mass spectral data were recorded using 6210 Time-ofFlight LC-MS system (Agilent Technologies, Santa Clara, CA, United States) connected to an Agilent 1200 Series HPLC instrument (Agilent Technologies, Waldbronn, Germany), with a degasser, a binary pump, an autosampler, a column compartment equipped with a Zorbax Eclipse XDB-C18 RRHT column $(1.8 \mu \mathrm{m}, 4.6 \mathrm{~mm} \times 50 \mathrm{~mm})$ and a diode-array detector, via ESI interface. The mobile phase consisted of water containing $0.2 \%$ formic acid (v/v; A) and acetonitrile (B). A gradient program was used as follows: $0-0.24 \mathrm{~min} 5 \% \mathrm{~B}, 0.24-10 \mathrm{~min}, 5-95 \% \mathrm{~B}$, 10-15 $\mathrm{min}, 95 \% \mathrm{~B}, 15-15.5 \mathrm{~min}, 95-5 \% \mathrm{~B}, 15.5-20 \mathrm{~min}, 5 \%$ B. The flow rate of mobile phase was $0.5 \mathrm{~mL} / \mathrm{min}$, the column temperature was $40^{\circ} \mathrm{C}$ and the injection volume was $10 \mu \mathrm{L}$. Spectral data from all the peaks were accumulated in the range of 190-900 nm. Full scan mass spectra were measured between 100 and $1500 \mathrm{~m} / \mathrm{z}$ in positive ion mode.

\section{Synthesis and Characterization of Bacterial Rhamnolipid Derivatives}

To a solution of di-rhamnolipids $(0.1 \mathrm{mmol}, 1$ eq. $)$ in $\mathrm{CH}_{2} \mathrm{Cl}_{2} / \mathrm{DMF}$ (2.7:0.3 mL) were added EDCI (0.12 mmol, 1.2 eq.), DMAP (0.05 mmol, 0.5 eq.) and amine (benzyl amine, piperidine and morpholine; $0.3 \mathrm{mmol}, 3$ eq.). The mixture was stirred for $16 \mathrm{~h}$ at room temperature. The mixture was diluted with water $(10 \mathrm{~mL})$ and extracted with $\mathrm{CH}_{2} \mathrm{Cl}_{2}(3 \times 15 \mathrm{~mL})$. The combined organic extract was dried with $\mathrm{MgSO}_{4}$, filtered and concentrated in vacuo. The residue was purified by flash column chromatography $\left(\mathrm{SiO}_{2}\right.$, ethyl acetate:methanol:dichloromethane in $4: 2: 1$ ratio) that afforded products as yellow thick oils.

To obtain silylated derivative to a solution of rhamnolipids ( $0.1 \mathrm{mmol}, 1$ eq.) in DMF (3 mL) TBDMS-Cl (1.6 mmol, 16 eq.) $\mathrm{AgNO}_{3}$ (1.6 mmol, 16 eq.) and pyridine (3.2 mmol, 32 eq.) were added. The mixture was stirred overnight at ambient temperature before it was diluted with water $(10 \mathrm{~mL})$ and extracted with ethyl acetate $(3 \times 20 \mathrm{~mL})$. The combined organic extract was dried with $\mathrm{MgSO}_{4}$, filtered and concentrated in vacuum to yield the product which was purified via silica gel flash chromatography using petroleum ether/EtOAc (7:1) as eluent to provide the title compound as a colorless oil.

Physico-chemical properties of all compounds were predicted using the Marvin Sketch 17.2.13.0 program (ChemAxon ${ }^{1}$ ). Consensus methods were used for calculating $\log \mathrm{P}$ and hydrophilic-lipophilic balance (HLB) values.

\section{Test Organisms}

Test organisms for the antibacterial assays were obtained from the National Collection of Type Cultures (NCTC) and the American Type Culture Collection (ATCC). P. aeruginosa PAO1 NCTC 10332, S. aureus ATCC 25923 and S. aureus ATCC 43300 (MRSA) and S. marcescens ATCC 27117 were used in this study. One clinical isolate of $P$. aeruginosa DM50 with high ability to form biofilms and resistance to metronidazole, clindamycin and amoxicillin was also included (Glisic et al., 2016). Bacterial strains were grown in Luria Bertani (LB) broth at $37^{\circ} \mathrm{C}$ on a rotary shaker at $180 \mathrm{rpm}$.

All rhamnolipids utilized for the bioactivity assessments in this study along with sources and descriptions are listed in Supplementary Table S1.

\section{Antimicrobial Susceptibility Tests for Planktonic Cells}

The minimum inhibitory concentration (MIC) of rhamnolipids and their derivatives were determined according to standard broth microdilution assays recommended by the National Committee for Clinical Laboratory Standards (M07-A8).

Stock solutions of rhamnolipids and derivatives were prepared in DMSO (50 $\left.\mathrm{g} \mathrm{L}^{-1}, \mathrm{w} / \mathrm{v}\right)$. The highest concentration used was $500 \mathrm{mg} \mathrm{L}^{-1}$. The inoculums were $10^{5}$ colony forming units (cfu) $\mathrm{mL}^{-1}$. The MIC value corresponds to the lowest concentration that inhibited the growth after $24 \mathrm{~h}$ at $37^{\circ} \mathrm{C}$ for $P$. aeruginosa and S. aureus or at $30^{\circ} \mathrm{C}$ for $S$. marcescens.

\section{Inhibition of Biofilm Formation}

Biofilm quantification assays were performed in 96-well microtiter plates using a crystal violet (CV) method to stain adherent cells (Merritt et al., 2005). Overnight cultures of bacteria were diluted to $5 \times 10^{7} \mathrm{cfu} \mathrm{mL}^{-1}$ in $\mathrm{LB}$ and $100 \mu \mathrm{L}$ was added to the wells in the presence of test compounds or DMSO $(0.1 \%, \mathrm{v} / \mathrm{v})$. Biofilms formed for $24 \mathrm{~h}$ at $37^{\circ} \mathrm{C}$ for P. aeruginosa and S. aureus, or $30^{\circ} \mathrm{C}$ for $S$. marcescens were washed and adherent cells stained with $\mathrm{CV} 0.1 \%(\mathrm{v} / \mathrm{v}) . \mathrm{BFIC}_{50}$ (concentration of compound that inhibited biofilm formation by $50 \%$ ) was determined for each compound.

The effect of rhamnolipids on $P$. aeruginosa biofilm formation was examined additionally by introducing $3 \mathrm{~h}$ adhesion phase

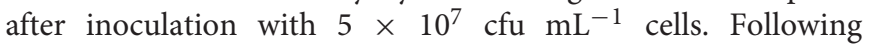
adhesion, the supernatant was removed and, after two washing

\footnotetext{
${ }^{1}$ http://www.chemaxon.com
} 
steps with phosphate buffered saline (PBS), test compounds or DMSO were applied and the biofilms were quantified after $24 \mathrm{~h}$ using CV. Each biofilm formation assay was performed in six wells and repeated at least three times.

\section{Scanning Electron Microscopy (SEM)}

To study the effect of di-rhamnolipids on $P$. aeruginosa PAO1 adherence to silicone surfaces and overnight bacterial culture was diluted to $5 \times 10^{7} \mathrm{cfu} \mathrm{mL} \mathrm{mL}^{-1}$ in $\mathrm{LB}$ and $2 \mathrm{ml}$ was added per well in six well microtiter plate. The silicone catheter pieces (Romed, Wilnis, Holland) of $1 \mathrm{~cm}$ were placed in each well containing diluted bacteria and incubated in the presence of di-rhamnolipids from Lysinibacillus sp. BV152.1 (F3) or DMSO. After $24 \mathrm{~h}$, the culture medium was removed and the catheters were washed three times in PBS in order to remove the non-adherent strains. Biofilms were then fixed with cold methanol and the samples dried before the examination.

Catheters were glued to double-sided conductive carbon tab stuck on standard vacuum-clean stub, and were coated with gold (thickness of 15-20 nm) by the sputtering process (Leica EM SCD005 sputtering machine, Leica Microsystems, Mannheim, Germany). Sputtering was performed in the vacuum chamber under pressure $<0.05$ mbar using sputter current of $40 \mathrm{~mA}$, the working distance of $50 \mathrm{~mm}$ and sputter time of 100 s. Such prepared samples were examined by JEOL JSM6610LV microscope (JEOL United States, Inc., Peabody, MA, United States). An acceleration voltage of $20 \mathrm{kV}$ was used.

\section{Biofilm Microscopy}

To study the effect of di-rhamnolipids on P. aeruginosa PAO1 adherence to glass or plastic surfaces biofilms were developed on cover slips and examined under the microscope. An overnight culture of $P$. aeruginosa PAO1 was diluted to $5 \times 10^{7} \mathrm{cfu} \mathrm{mL}^{-1}$ in $\mathrm{LB}$ and $2 \mathrm{ml}$ was added per well of six well microtiter plate containing glass or plastic cover slips. After $24 \mathrm{~h}$, non-adherent cells were removed and biofilms were washed with $0.9 \% \mathrm{NaCl}$ and stained with $2.5 \mu \mathrm{M}$ SYTO9 green fluorescent dye and $2.5 \mu \mathrm{M}$ propidium iodide (PI) red fluorescent dye of Live/Dead staining kit (LIVE/DEAD ${ }^{\circledR}$ BacLight $^{\mathrm{TM}}$ Bacterial Viability Kit, Thermo Fisher Scientific, Waltham, MA, United States). Cells were observed under a fluorescence microscope (Olympus BX51, Applied Imaging Corp., San Jose, CA, United States) under $100,000 \times$ magnification (glass) or $40,000 \times$ magnification (plastic).

\section{Anti-adhesion Assay}

Anti-adhesion assay was performed as previously described (Shetye et al., 2014) with some modifications. An overnight culture of $P$. aeruginosa PAO1 containing pBBR2-GFP (da Silva et al., 2014) was subcultured (initial OD600 = 0.02) in M9+/LB (95:5) containing gentamycin $\left(80 \mathrm{mg} \mathrm{L}^{-1}\right)$, tetracycline $(20 \mathrm{mg}$ $\left.\mathrm{L}^{-1}\right)$, and chloramphenicol $\left(50 \mathrm{mg} \mathrm{L}^{-1}\right)$ at $37^{\circ} \mathrm{C}$ in a rotary shaker $(200 \mathrm{rpm})$. After reaching OD600 $=0.1$, aliquots $(200 \mu \mathrm{L})$ were transferred to the wells of black polystyrene microtiter plate with rhamnolipids, derivatives or DMSO (control). After incubation at $37^{\circ} \mathrm{C}$ for $2 \mathrm{~h}$, bacterial cultures were discarded, and the fluorescence of adherent cells was measured after addition of fresh M9+/LB (95:5) medium using Tecan Infinite 200 Microplate reader (Tecan Group Ltd., Switzerland, lex $=500 \mathrm{~nm}$, lem $=540 \mathrm{~nm}$ ). Background signal (M9+/LB (95:5) was subtracted from all the samples. Assays were repeated three times; inhibition values are the averages of six replicate wells from one experiment.

\section{Biofilm Dispersion Assay}

An overnight culture of $P$. aeruginosa was diluted to $5 \times 10^{7}$ cfu $\mathrm{mL}^{-1}$ in LB and $100 \mu \mathrm{L}$ was added to the wells in 96-well microtiter plates and incubated for $24 \mathrm{~h}$ at $37^{\circ} \mathrm{C}$. After removal of the supernatant and two washing steps with PBS adherent cells were treated with different concentrations of rhamnolipids or derivatives for additional $24 \mathrm{~h}$ and the biofilms were quantified using $\mathrm{CV}$ as described above. Biofilm dispersion assays were performed in six wells and repeated three times.

\section{Cytotoxicity Assay}

MRC5 human lung fibroblasts were obtained from the ATCC. Cells were maintained as monolayer cultures in RPMI-1640 supplemented with $100 \mathrm{mg} \mathrm{L}^{-1}$ streptomycin, $100 \mathrm{U} \mathrm{mL}^{-1}$ penicillin and 10\% (v/v) FBS (all from Sigma, Munich, Germany). Cells were grown in a humidified atmosphere of 95\% air and 5\% $\mathrm{CO}_{2}$ at $37^{\circ} \mathrm{C}$.

Cytotoxicity on MRC5 cells was evaluated with 3-(4,5dimethylthiazol-2-yl)-2,5-diphenyltetrazolium bromide (MTT). The assay was carried out after $48 \mathrm{~h}$ of cell incubation in the media containing test compounds at different concentrations and the viability was measured as described (Mihajlovic et al., 2012). The results are presented as the percentage of the control (untreated cells) that was arbitrarily set to $100 \%$.

\section{Statistical Analysis}

The results were analyzed by Student's $t$-test using SPSS version 20 software. A $P$-value lower than 0.05 was considered as statistically significant.

\section{RESULTS}

\section{Isolation and Identification of BV152.1}

The rhizosphere of medicinal plant G. hederacea L. (ground ivy) proved a prolific source of aerobic bacteria with 32 morphologically differing isolates collected from this source (data not shown). Culture extracts from these isolates were screened for their ability to inhibit biofilm formation in $P$. aeruginosa PAO1 using CV based assay in 96-well polystyrene plates. During the preliminary screening, the crude cell extract of the isolate BV152.1 showed the most potent ability to inhibit $60 \%$ of biofilm formation when applied in the concentration of $500 \mu \mathrm{g}$ $\mathrm{mL}^{-1}$ while no bactericidal activity of the extract was observed. This isolate was selected for structural characterization of its secondary metabolites as the most promising one. The isolate BV152.1 was identified as a member of the Gram-positive genus Lysinibacillus on the basis of 16S rRNA gene sequence analysis. Its 16S rRNA gene sequence (GenBank access No. KY933395) showed $99 \%$ similarity with type strains of Lysinibacillus louembei 
NM73 (NR_145586.1), L. meyeri WS 4626 (NR_117577.1), and L. odysseyi YH2 (KM873372.1).

\section{Di-Rhamnolipids As Active Components of Lysinibacillus sp. BV152.1 Culture Extracts}

In order to identify the component with biofilm formation inhibitory activity produced by Lysinibacillus sp. BV152.1, 1 L culture grown for 7 days at $30^{\circ} \mathrm{C}$ was extracted with ethyl acetate and the total mass of $800 \mathrm{mg}$ crude cell extract was obtained. The crude cell extract was subsequently fractionated, the activity of each fraction measured and the fraction designated as F3 was identified as the most active one. After further purification, the total mass of $\mathrm{F} 3$ was determined to be $60 \mathrm{mg}$ and it showed 50\% inhibition of biofilm formation when applied in a concentration of $50 \mu \mathrm{g} \mathrm{mL}^{-1}$ (Table 1).

${ }^{1} \mathrm{H}$-NMR and ${ }^{13} \mathrm{C}$-NMR spectra of $\mathrm{F} 3$ showed the presence of fatty acid chains and carbohydrate moieties (Figure 1A) indicative of the characteristic spectra of bacterial dirhamnolipids. The peak observed at $\delta 0.88 \mathrm{ppm}$ indicated the presence of terminal methyl group and the peak at $\delta 1.26$, characteristic for methylene group, indicated the presence of a straight-chain fatty acid. The peaks observed at $\delta 4.15-3.36$ and peaks for two anomeric protons at $\delta 4.90$ confirmed the presence of two carbohydrate units. The ${ }^{13} \mathrm{C}-\mathrm{NMR}$ analysis demonstrated two carbonyl peaks at $\delta 173.7$ and 171.4, two anomeric carbon peaks at $\delta 102.5$ and 94.5 and expected number of peaks for carbohydrate and fatty acid units. In general, the spectral characteristics obtained for F3 (provided in Supplementary material) were in good agreement with the data published in the literature for di-rhamnolipids (Sharma et al., 2007).

The exact lengths of the fatty acid chains were deduced from HPLC-MS data (Figure 1B). Sodiated molecular ions, $[\mathrm{M}+\mathrm{Na}]+$, in the ESI+MS data were observed at $\mathrm{m} / z 701$ (Rha-Rha-C10-C12, 11.16 min, 9\%), 699 (Rha-Rha-C10-C12:1,
$10.68 \mathrm{~min}, 8 \%$ ), 673 (Rha-Rha-C10-C10, 10.03 min, 63\%, major), and 645 (Rha-Rha-C8-C10, 8.96 min, 17\%), 527 (Rha-C10-C10, $10.86 \mathrm{~min}, 3 \%)$.

Considering the identified structural characteristics of F3, we have selected commercially available $P$. aeruginosa sp. derived rhamnolipids (R90), as the model and the control compound. R90 was determined to contain mono- vs. di-rhamnolipid form in 4:1 ratio (AGAE Technologies, Figure 1C) with the major component of Rha-Rha-C10-C10 in more than $90 \%$.

\section{Anti-biofilm Properties of Di-Rhamnolipids}

Di-rhamnolipids from Lysinibacillus sp. BV152.1 (F3) containing mainly di-rhamnolipids with Rha-Rha-C10-C10 63\% showed dose-dependent anti-biofilm formation activity in $P$. aeruginosa PAO1 with $50 \mu \mathrm{g} \mathrm{mL}^{-1}$ determined as $\mathrm{BFIC}_{50}$ (Figure 2A). In order to assess whether di-rhamnolipids from Lysinibacillus sp. BV152.1 affected biofilm growth, $P$. aeruginosa PAO1 cells were left to attach to the surface of microtiter wells for $2 \mathrm{~h}$ prior to incubation with F3 for $24 \mathrm{~h}$. Inhibition of biofilm formation was similar in the presence or absence of the cell adhesion phase showing that di-rhamnolipids affected both cell attachment and biofilm growth (Figure 2A).

Anti-biofilm properties of di-rhamnolipids from Lysinibacillus sp. BV152 were compared to that of commercially available rhamnolipids obtained by fermentation of P. aeruginosa sp. (R90) (Figure 2B). Di-rhamnolipids (di-Rha) from this sample were also purified and used for the comparison. Rhamnolipids mixture (R90) and purified di-Rha from $P$. aeruginosa showed slightly lower activity against $P$. aeruginosa $\mathrm{PAO} 1$ biofilm formation $\left(\right.$ BFIC $_{50}=75 \mu \mathrm{g} \mathrm{mL}^{-1}$ ) comparing to F3. The effects of R90 and di-Rha on biofilm formation were comparable when the treatments were applied without cell adhesion phase. However, when bacteria were left to attach to the surface prior to treatments, di-Rha stimulated biofilm formation when added in concentrations up to $50 \mu \mathrm{g} \mathrm{mL}^{-1}$. Di-rhamnolipids from

TABLE 1 | Antibacterial activity of rhamnolipids mixture (R90), di-rhamnolipids and di-rhamnolipid derivatives from Lysinibacillus sp. BV152.1 and Pseudomonas aeruginosa determined after $24 \mathrm{~h}$ incubation.

\begin{tabular}{|c|c|c|c|c|c|}
\hline Rhamnolipids $\mathrm{MIC}^{\mathrm{a}}\left(\mu \mathrm{g} \mathrm{mL}^{-1}\right)$ & P. aeruginosa PAO1 & P. aeruginosa DM50 & S. aureus ATCC 25923 & S. aureus MRSA & S. marcescens ATCC 27117 \\
\hline \multicolumn{6}{|l|}{ Lysinibacillus sp. BV152.1 } \\
\hline di-Rha mixture (F3) & $>500$ & $>500$ & $>500$ & $>500$ & $>500$ \\
\hline di-Rha-Bn & $>500$ & $>500$ & $>500$ & $>500$ & $>500$ \\
\hline di-Rha-Pip & $>500$ & $>500$ & $>500$ & 250 & $>500$ \\
\hline di-Rha-Mor & $>500$ & $>500$ & $>500$ & $>500$ & $>500$ \\
\hline di-rha-TBDMS & $>500$ & $>500$ & $>500$ & $>500$ & $>500$ \\
\hline \multicolumn{6}{|l|}{ P. aeruginosa } \\
\hline R90 & $>500$ & $>500$ & 250 & 250 & $>500$ \\
\hline di-Rha & $>500$ & $>500$ & $>500$ & $>500$ & $>500$ \\
\hline di-Rha-Bn & $>500$ & $>500$ & 62.5 & 125 & $>500$ \\
\hline di-Rha-Pip & $>500$ & $>500$ & 62.5 & 62.5 & $>500$ \\
\hline di-Rha-Mor & $>500$ & $>500$ & 62.5 & 62.5 & $>500$ \\
\hline di-Rha-TBDMS & $>500$ & $>500$ & $>500$ & $>500$ & $>500$ \\
\hline
\end{tabular}

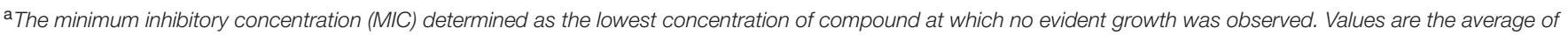
two independent experiments performed in four wells. 


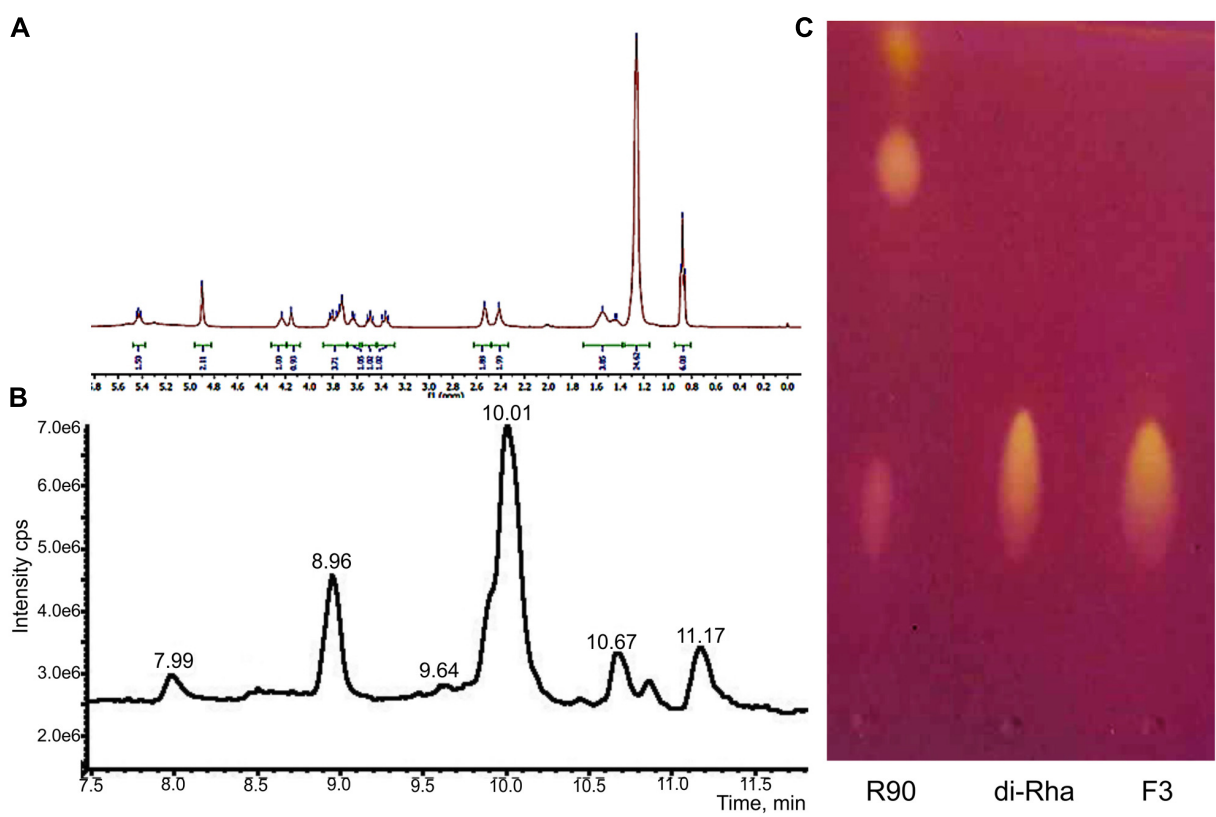

FIGURE 1 | Structural characterization of di-rhamnolipids (F3) isolated from Lysinibacillus sp. BV152.1. (A) ${ }^{1} \mathrm{H}$ NMR spectrum of F3, (B) HPLC chromatogram of F3, and $\mathbf{( C )}$ thin layer chromatography of Pseudomonas aeruginosa rhamnolipids (R90), purified di-rhamnolipids from R90 (di-Rha) and di-rhamnolipids fraction isolated from Lysinibacillus sp. BV152.1 culture (F3).
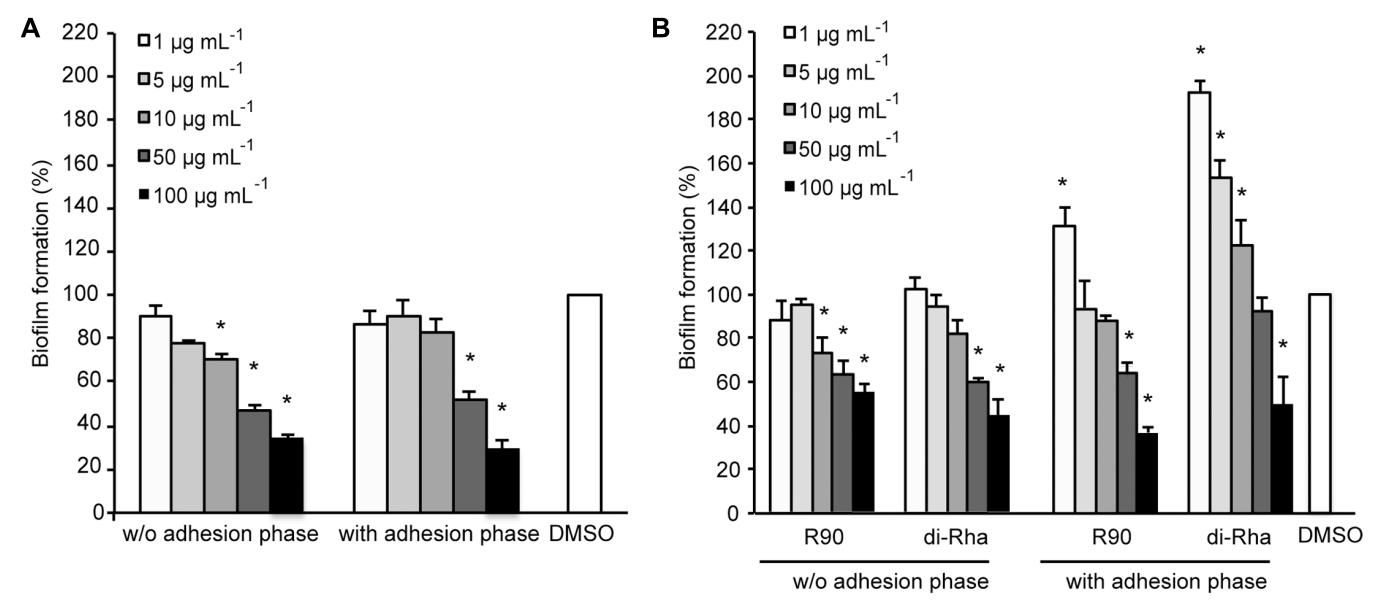

FIGURE 2 | Inhibition of P. aeruginosa PAO1 biofilm formation with (A) di-rhamnolipids produced by Lysinibacillus sp. BV152.1 (F3) and (B) P. aeruginosa (rhamnolipids mixture and purified di-Rha). ${ }^{*} P<0.05$.

both sources showed an equal potency in inhibition of biofilm formation at concentrations above $50 \mu \mathrm{g} \mathrm{mL}^{-1}$.

Di-rhamnolipids from Lysinibacillus sp. BV152.1 efficiently inhibited bacterial adhesion to the polystyrene surface of microtiter plates and also to the surface of silicone catheters or glass coverslips as visualized by SEM and fluorescent microscopy, respectively (Figure 3).

Production of rhamnolipids in $P$. aeruginosa enables its swarming motility (Caiazza et al., 2005). Therefore, the effect of di-rhamnolipids on $P$. aeruginosa PAO1 swarming motility was analyzed next and the results showed that both F3 and R90 stimulated swarming in dose-dependent manner (Supplementary Figure S1). The observed effect was more prominent in the presence of di-rhamnolipids from Lysinibacillus sp. BV152.1.

\section{Derivatization of Di-Rhamnolipids and Their Antibacterial and Cytotoxic Properties}

The semi-synthetic approach of generating amide derivatives from two different sources of di-Rha was straightforward, with products obtained in high purity and yields from 25 to 55\% 

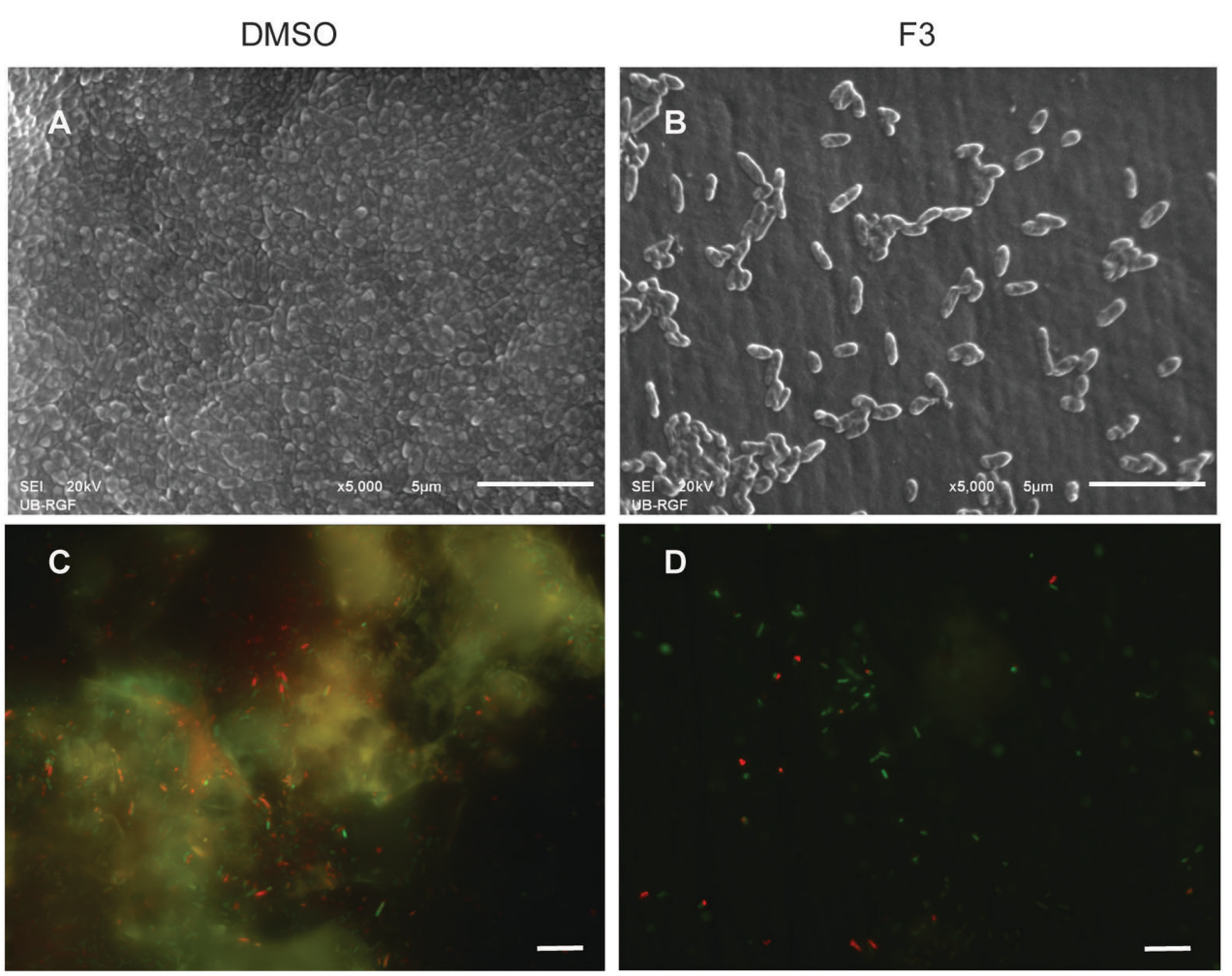

FIGURE 3 | Inhibition of cell attachment and biofilm formation with di-rhamnolipids from Lysinibacillus sp. BV152.1. Biofilms P. aeruginosa PAO1 were formed for $24 \mathrm{~h}$ on silicone catheter $(\mathbf{A}, \mathbf{B})$ or glass $(\mathbf{C}, \mathbf{D})$ in the presence of DMSO $(0.1 \%)$ or F3 $\left(50 \mu \mathrm{gL}^{-1}\right)$. Biofilms were analyzed by scanning electron microscopy $(\mathrm{SEM}$; $\mathbf{A}, \mathbf{B})$ or fluorescent microscopy (C,D). In (C,D) bacteria labeled with Syto9 appeared green and bacteria stained with propidium iodide (PI) are red, scale bars represent $10 \mu \mathrm{m}$.

(Figure 4). Products were characterized using NMR and LC-MS (Supplementary Figures S2-S5). In addition, silylated derivative (di-Rha-TBDMS) was also generated for the control purposes. Physico-chemical parameters for all compounds were calculated revealing the HLB value ranging from 8.72 to 11.09 for di-Rha and amide derivatives, while this value for di-Rha-TBDMS was 5.35 (Figure 4). It is known that compounds with HLB 7-11 are considered wetting agents and water in oil emulsifiers, while the ones having HLB between 4 and 6 as water in oil emulsifiers (Pasquali et al., 2008; Muller et al., 2012). pKa was three times higher for amide derivatives in comparison to di-Rha and diRha-TBDMS indicating that they were weaker acids. $\log \mathrm{P}$ was between 3.36 and 5.07 for di-Rha and amide derivatives, while this value for di-Rha-TBDMS was 3-5 times higher, suggesting higher hydrophilicity of amide derivatives (Figure 4). Spectral data for the new compounds are provided in Supplementary material.

Antibacterial activity of all derivatives obtained from both rhamnolipids sources against $P$. aeruginosa $\mathrm{PAO} 1, P$. aeruginosa DM50, S. aureus ATCC 25923, S. aureus MRSA and S. marcescens was addressed. Neither F3 nor di-Rha derivatives obtained from Lysinibacillus sp. BV152.1 exhibited antibacterial activity at concentrations up to $500 \mu \mathrm{g} \mathrm{mL} \mathrm{mL}^{-1}$ against any of the bacterial species tested (Table 1). None of the derivatives synthetized from $P$. aeruginosa di-Rha affected $P$. aeruginosa and $S$. marcescens growth. However, $P$. aeruginosa derivatives diRha-Pip and di-Rha-Mor exhibited bactericidal activity against S. aureus ATCC 25923 strain and S. aureus MRSA with MIC concentrations $62.5 \mu \mathrm{g} \mathrm{mL}^{-1}$. Derivative Rha-Bn from $P$. aeruginosa demonstrated the same antibacterial activity against S. aureus MRSA.

Cytotoxicity of rhamnolipids mixture (R90), di-Rha mixture (F3), purified di-Rha and their derivatives was analyzed (Figure 5). Viability of human lung fibroblasts (MRC5) was not affected in the presence of F3, di-Rha and di-Rha-TBDMS when applied in concentrations up to $100 \mu \mathrm{g} \mathrm{mL}-1$. Rhamnolipids mixture R90 showed cytotoxic effect with $\mathrm{IC}_{50}$ value $50 \mu \mathrm{g} \mathrm{mL}^{-1}$. Derivatization of di-Rha substantially increased their cytotoxicity reaching almost $100 \%$ cells killing at concentrations of $25 \mu \mathrm{g}$ $\mathrm{mL}^{-1}$. These concentrations were 2.5 -fold lower than their MIC concentration values against $S$. aureus strains (Table 1) and potentially are limiting factor in their further development as antibiotics.

\section{Anti-biofilm Activity of Di-Rhamnolipids and Their Amide Derivatives}

Anti-biofilm properties of di-rhamnolipids and their derivatives were examined by addressing their influence on biofilm formation, cell adhesion and disruption of pre-formed biofilms. Biofilm formation in $P$. aeruginosa PAO1 was quantified 


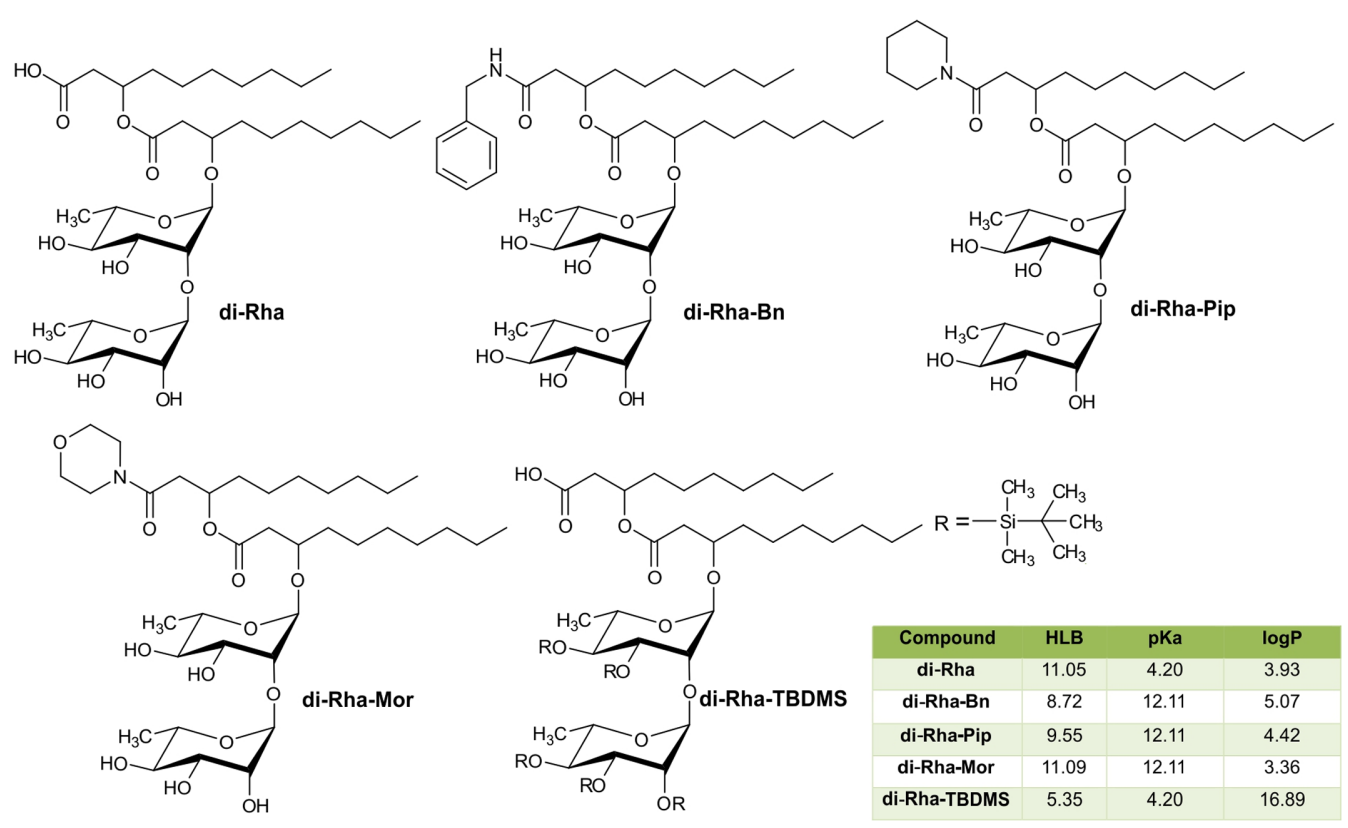

FIGURE 4 | Chemical structures of di-Rha (C10-C10) and amide derivatives synthesized in this study with calculated parameters hydrophilic-lipophilic balance (HLB), acid dissociation constant (pKa), partition coefficient (logP).

in the presence or absence of di-rhamnolipids and their derivatives. Derivative di-Rha-Mor from Lysinibacillus sp. BV152.1 showed increased anti-biofilm formation activity

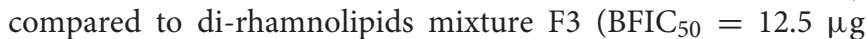
$\mathrm{mL}^{-1}$ ) inhibiting $90 \%$ biofilm formation at $100 \mu \mathrm{g} \mathrm{mL} \mathrm{m}^{-1}$ (Figures 6A, 7). Anti-biofilm formation activity of di-Rha$\mathrm{Bn}$ and di-Rha-Pip was twofold lower than that of F3 $\left(\mathrm{BFIC}_{50}=100 \mu \mathrm{g} \mathrm{mL}^{-1}\right)$. Amide derivatization of di-Rha from $P$. aeruginosa significantly improved activities of all derivatives with BFIC $_{50}=10 \mu \mathrm{g} \mathrm{mL}^{-1}$ (Figures 6B, 7). However, maximum activity was reached already at $50 \mu \mathrm{g} \mathrm{mL} \mathrm{m}^{-1}$ with biofilm formation inhibition up to $60 \%$. Derivatives di-Rha-TBDMS from both sources, showed no anti-biofilm formation activity.

To measure anti-adhesion activity of di-rhamnolipids and their amide derivatives, GFP fluorescence of $P$. aeruginosa PAO1GFP was used to quantify bacterial adhesion $2 \mathrm{~h}$ after inoculation. Relative to the amount of adhered bacteria without treatment (containing 0.1\% DMSO), derivatives di-Rha-Bn and di-Rha-Pip from Lysinibacillus sp. BV152.1 inhibited PAO1-GFP adhesion by $50 \%$ at concentrations $10 \mu \mathrm{g} \mathrm{mL}^{-1}$, while di-Rha-Mor showed $50 \%$ adhesion inhibition at $50 \mu \mathrm{g} \mathrm{mL}^{-1}$ (Figure 6C). Again, derivatives from $P$. aeruginosa exhibited less prominent antiadhesive activities inhibiting $P$. aeruginosa PAO1-GFP adhesion up to $35 \%$ at concentrations $100 \mu \mathrm{g} \mathrm{mL} \mathrm{mL}^{-1}$ (Figure 6D). Derivative di-Rha-TBDMS showed no significant anti-adhesion activity. Importantly, di-Rha and their derivatives did not affect GFP expression nor quenched its fluorescence (Supplementary Figure S10).

Potential to disperse formed biofilm is often more relevant to medical applications, and more challenging than inhibition of biofilm formation. Therefore, the ability of di-rhamnolipids and their amide derivatives to disperse $24 \mathrm{~h}$ old $P$. aeruginosa biofilms was examined next (Figures 6E,F, 8). Di-rhamnolipids from Lysinibacillus sp. BV152.1 (F3) were more effective in biofilm disruption with BDIC $_{50} 10 \mu \mathrm{g} \mathrm{mL}^{-1}$ (BDIC $_{50}$ is the concentration of compound that caused $50 \%$ biofilm disruption) comparing to di-Rha from $P$. aeruginosa which disrupted biofilms up to $20 \%$ at concentrations $100 \mu \mathrm{g} \mathrm{mL} \mathrm{m}^{-1}$. The strongest biofilm dispersion activity was measured for diRha-Mor derivatives from both sources, with BDIC $_{50}$ values 12.5 and $50 \mu \mathrm{g} \mathrm{mL}^{-1}$ from Lysinibacillus sp. BV152.1 and $P$. aeruginosa, respectively. Both di-Rha-Mor derivatives were able to disrupt more than $80 \%$ of pre-formed biofilms when applied at $100 \mu \mathrm{g} \mathrm{mL}^{-1}$.

Taken together, these results demonstrated that amide derivatization improved di-rhamnolipids anti-biofilm properties, with di-Rha-Mor derivative being the most active compound. The results were confirmed by comparing anti-biofilm formation activities of di-rhamnolipids and their amide derivatives against different bacterial species (Tables 2, 3). The improvement of rhamnolipids anti-biofilm formation activity with amide derivatization was more prominent with di-rhamnolipids from Lysinibacillus sp. BV152.1. Anti-biofilm formation activity of these derivatives was species specific with the highest activity observed for di-Rha-Bn and di-Rha-Pip against S. aureus ATCC 25923 (>90\% inhibition). All three derivatives were active against S. marcescens biofilm formation ( $>80 \%$ ), while the highest activity against $P$. aeruginosa biofilms was observed for di-Rha-Mor inhibiting $80 \%$ biofilm formation. The most active $P$. aeruginosa di-Rha derivative was also di-Rha-Mor, with improved anti-biofilm formation activity compared to di-Rha against S. aureus and S. marcescens (70\% and 85\% 

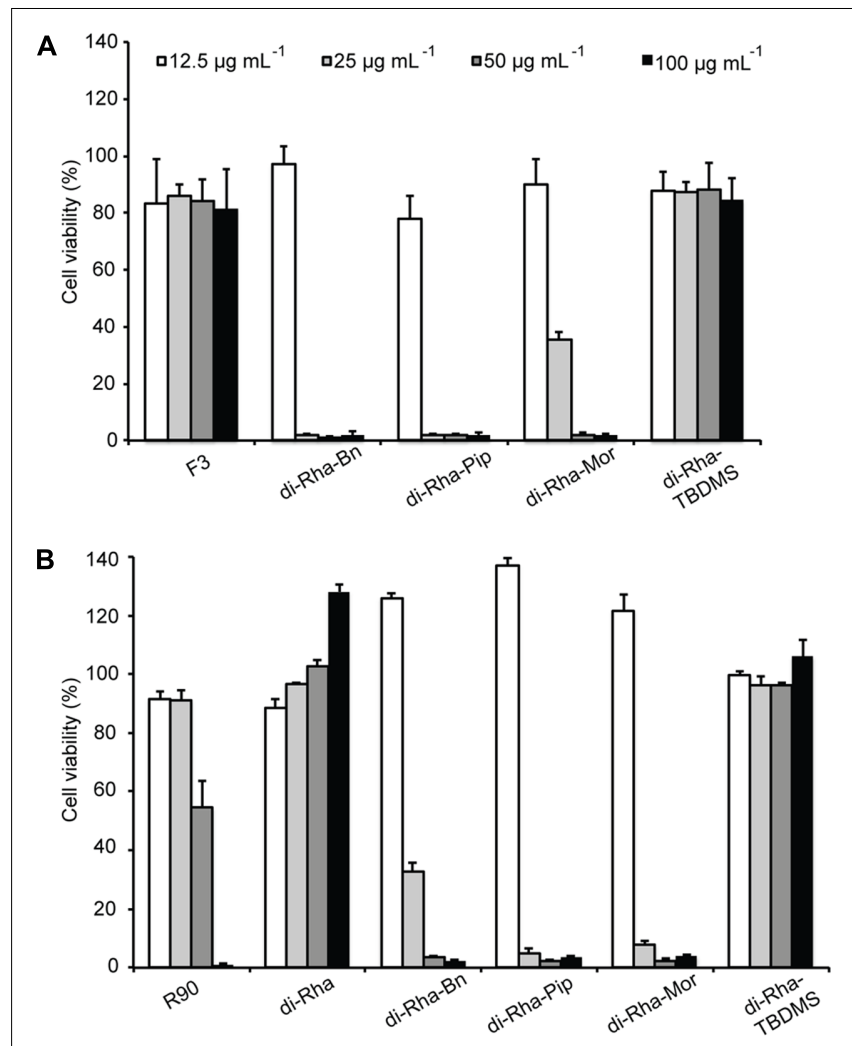

FIGURE 5 | Cytotoxic effects of rhamnolipids mixtures, di-rhamnolipids and their derivatives from Lysinibacillus sp. BV152.1 (A) and P. aeruginosa (B) on human fibroblasts (MRC5) measured by MTT method, following $48 \mathrm{~h}$ exposure. Values are representative of two independent experiments $\pm S D$.

inhibition at $50 \mu \mathrm{g} \mathrm{mL}^{-1}$, respectively). The strongest inhibition of $P$. aeruginosa PAO1 biofilm formation was achieved with $P$. aeruginosa di-Rha-Bn and di-Rha-Pip treatments, while none of the derivatives affected biofilm formation in clinical isolate $P$. aeruginosa DM50 (Table 2). Derivatization of dirhamnolipids from both sources also improved their biofilm dispersion activity against $P$. aeruginosa DM50, S. aureus ATCC 25923 and S. aureus MRSA (Table 3). Dispersion of S. marcescens biofilms was efficient only with di-Rha-Bn and di-Rha-Mor from Lysinibacillus sp. BV152.1 (63\% and 58\% biofilm dispersion at $50 \mu \mathrm{g} \mathrm{mL}^{-1}$, respectively). Di-Rha from both sources and the other derivatives showed no biofilm dispersion activity against this bacterium, most likely be due to slightly different carbon chain length between di-rhamnolipid sources.

\section{DISCUSSION}

Rhamnolipids are amphiphilic glycolipids biosynthesized by bacteria that, due to their low toxicity and biodegradability, are potential replacements for synthetic surfactants. For $P$. aeruginosa, secretion of rhamnolipids is critical for biofilm formation, dispersion of bacteria from the mature biofilms and for their swarming motility (Davey et al., 2003; Nickzad and
Deziel, 2014; Wittgens et al., 2016). Rhamnolipids are mainly produced by species of $P$. aeruginosa, ubiquitous opportunistic pathogen, which makes the isolation of novel safer natural producers an important task. In the present study, during an effort of identification of novel bacterial secondary metabolites with anti-biofilm activity, a new rhamnolipid producing bacterial strain has been isolated from the plant rhizosphere and identified as Lysinibacillus sp. BV152.1. Members of this Gram-positive genus, with the ability to form endospores under harsh environmental conditions, have been isolated from various environments, however, they are often found in soil and in association with plants (Ouoba et al., 2015; Fredsgaard et al., 2017; Govindasamy et al., 2017; Yan et al., 2017). L. fusiformis S9, isolated from the river bank soil sample in India, has been reported to produce glycolipids with surfactant properties, but the identity of the sugar moiety was not confirmed and in contrast to BV152.1 isolate, the unsaturated alkanoic acid was predominant lipid chain (Pradhan et al., 2014). Therefore this is the first report that undoubtedly confirmed the production of rhamnolipids by a strain belonging to genus Lysinibacillus. Some bacteria are known to produce only mono-rhamnolipids, while some produce mono- and di-rhamnolipids in various ratios, depending on the culture conditions. Here, anti-biofilm activity guided fractionation and purification of the bacterial culture extract lead toward the identification of pure di-Rha fraction (Figure 1).

It is well documented that rhizosphere microorganisms show wide ability to produce secondary metabolites with the pronounced antagonistic activity toward plant pathogens (Compant et al., 2005). Other bacterial isolates from the plant rhizosphere producing rhamnolipids have been isolated, however, they mostly belonged to Pseudomonas genus (Sharma et al., 2007). Rhamnolipid production level of Pseudomonas sp. GRP3 was comparable to production of di-Rha in Lysinibacillus sp. BV152.1 (0.041 vs. $0.06 \mathrm{~g} \mathrm{~L}^{-1}$ ), however, Pseudomonas sp. GRP3 mainly produced mono-Rha. The di-rhamnolipid fraction of this strain had similar composition to Lysinibacillus sp. BV152.1, with Rha-Rha-C10-C10 being the most prominent (87\%), followed by Rha-Rha-C8-C10 and Rha-Rha-C10-C12 (Sharma et al., 2007). In general, the principal rhamnolipids considered to be produced by $P$. aeruginosa are Rha-C10C10 and Rha-Rha-C10-C10 (Maier and Soberón-Chávez, 2000; Muller et al., 2010). Rhamnolipids can be commercially produced by $P$. aeruginosa at the level of $100 \mathrm{~g} \mathrm{~L}^{-1}$, upon extensive optimizations of fermentation conditions (Maier and SoberónChávez, 2000; Muller et al., 2012; Dobler et al., 2016). The fatty acid chain may vary from 8 to 14 carbon molecules (Abdel-Mawgoud et al., 2010). However, it was shown that biotechnologically obtained $\beta$-hydroxy alkanoic, of this carbon chain range, acids have moderate antimicrobial activity, but can be a good platform for synthesis of non-toxic molecules with improved antimicrobial properties (Radivojevic et al., 2016).

Biosurfactants, including rhamnolipids, affect the initial attachment on various surfaces, thus help prevention of the biofilm formation (Neu, 1996; Vatsa et al., 2010; Sodagari et al., 2013; Zhong et al., 2015). It was demonstrated that rhamnolipids (mixture of Rha-C10-C10 and di-Rha-C10-C10) 
A

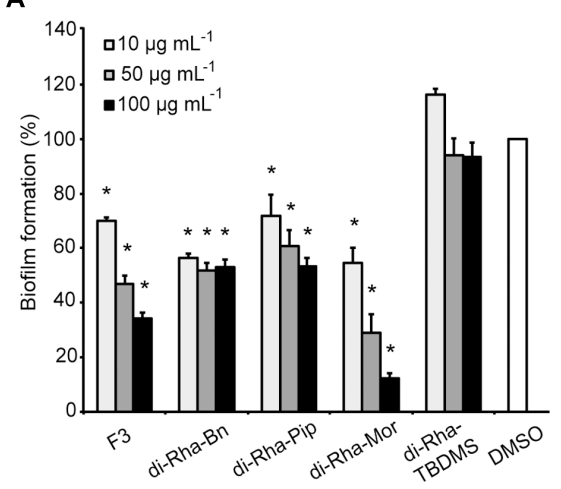

C

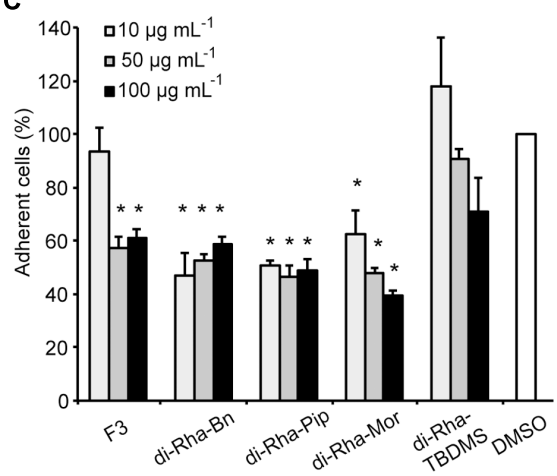

E

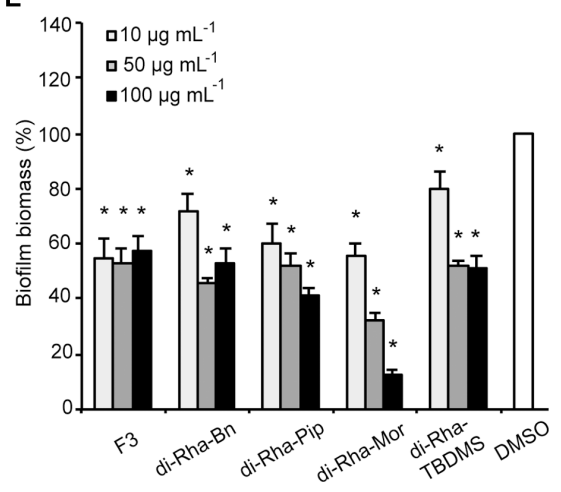

B

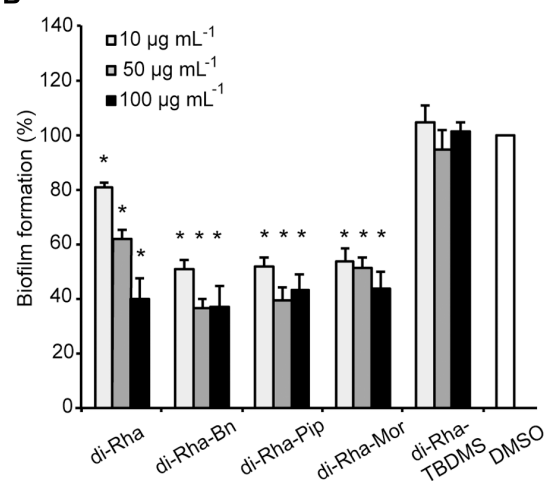

D

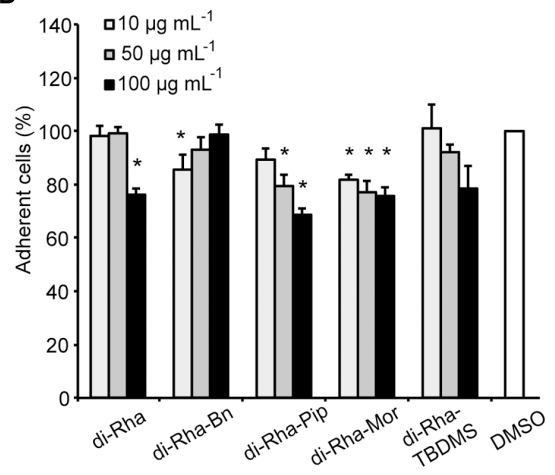

$\mathbf{F}$

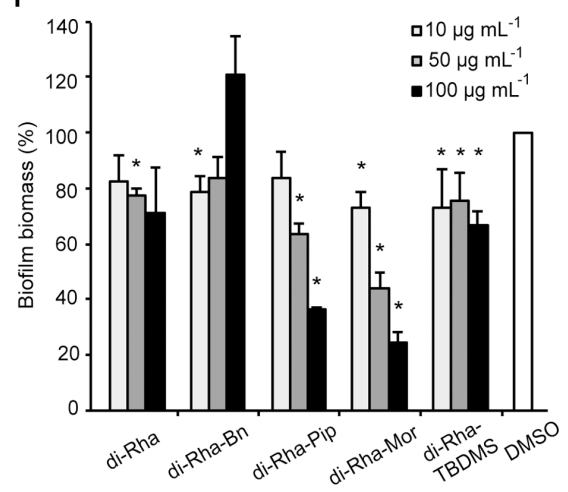

FIGURE 6 | Pseudomonas aeruginosa PAO1 biofilm formation (A,B), cell adhesion (C,D), or biofilm disruption (E,F; \%) in the presence of di-rhamnolipids isolated from Lysinibacillus sp. BV152.1 (A,C,E) or $P$. aeruginosa (B,D,F) and their derivatives. Values are presented as mean \pm SD. ${ }^{*} P<0.05$.

could prevent the attachment of $P$. aeruginosa, $P$. putida, and Escherichia coli, as well as S. epidermidis and Bacillus subtilis on glass and octadecyltrichlorosilane modified hydrophobic glass in concentration range from 10 to $200 \mu \mathrm{g} \mathrm{mL}-1$ to various extent (Sodagari et al., 2013). Inhibition of microbial growth, as well as a change of cell surface hydrophobicity was examined as a potential mechanism for this activity, but the responsible mechanism of the observed effect remained unknown. In the case of di-Rha utilized in this study, the antimicrobial effect has not been observed for concentrations up to $500 \mu \mathrm{g} \mathrm{mL}^{-1}$, while from the comparison of anti-biofilm activity with and without cell adhesion phase, it can be concluded that at concentrations of $50 \mu \mathrm{g} \mathrm{mL} \mathrm{m}^{-1}$ and above di-Rha reduced cell adhesion, while at lower concentrations affected biofilm maturation. Similarly, glycolipids from L. fusiformis S9 were found to inhibit bacterial attachment and caused the complete inhibition of E. coli and Streptococcus mutans biofilm formation at $40 \mu \mathrm{g} \mathrm{mL}^{-1}$ without affecting their growth (Pradhan et al., 2014). Di-Rha from both sources efficiently inhibited biofilm formation on different microorganisms including two antibiotic-resistant $S$. aureus MRSA and clinical isolate $P$. aeruginosa DM50 strains and were efficient on two types of surfaces, silicone catheter and glass. Both of these findings have great value for the possible future applications in biomedicine.

Previously limited access to relatively pure rhamnolipid materials at the gram scale has hindered extensive 

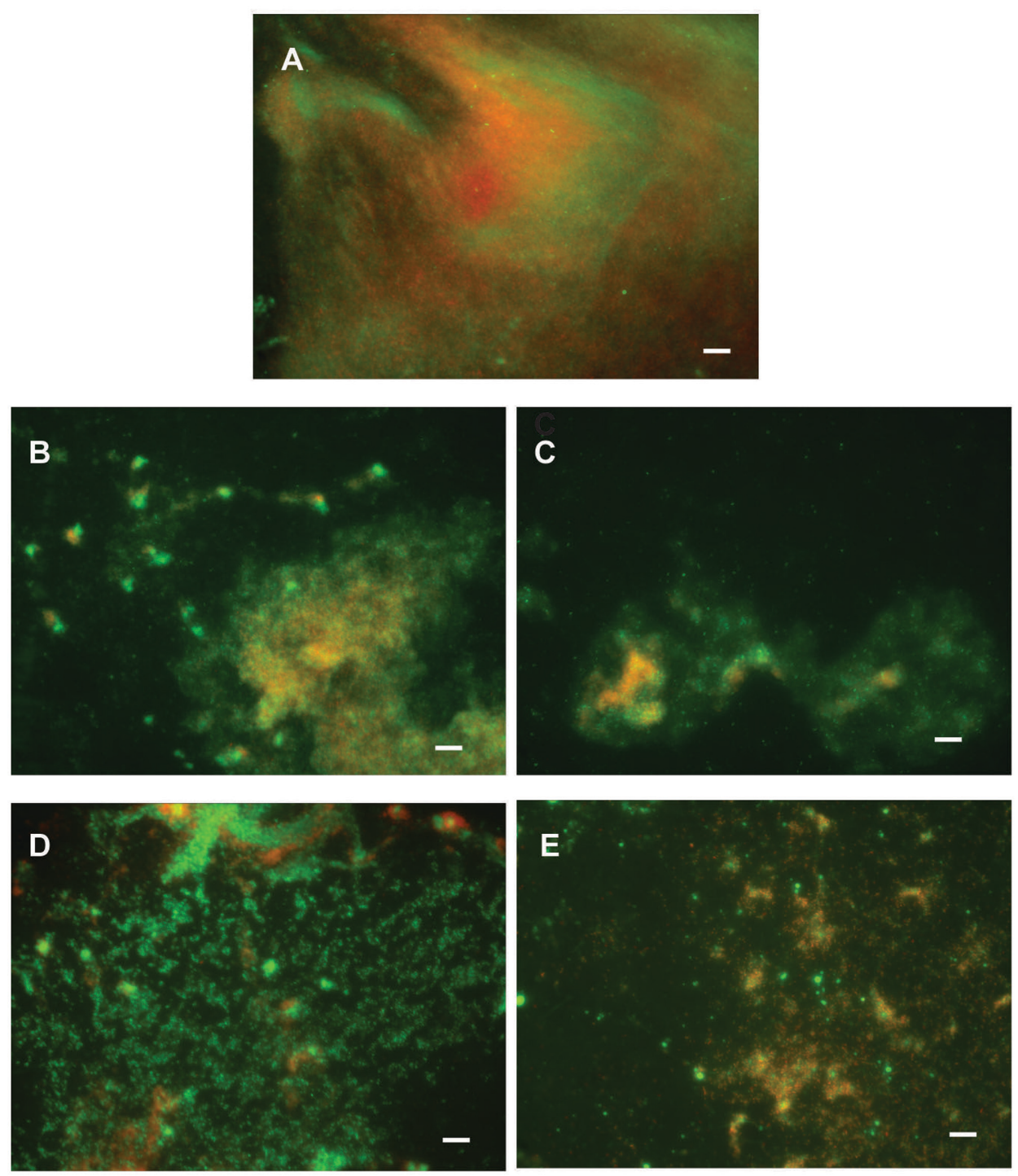

FIGURE 7 | Pseudomonas aeruginosa PAO1 biofilm formation on plastic surfaces in the presence of 0.1\% DMSO (A), F3 (B), di-Rha-Mor derivative from Lysinibacillus sp. BV152.1 (C), or di-Rha (D), and di-Rha-Mor derivative from P. aeruginosa (E) at $50 \mu \mathrm{g} \mathrm{mL}{ }^{-1}$. Biofilms were stained with Syto9 (green) and PI (red), scale bars represent $10 \mu \mathrm{m}$.

characterization of rhamnolipid structure-activity relation. Here, an efficient semi-synthetic methodology has been applied to the mixture and pure di-Rha, yielding three amide derivatives that were prepared and subsequently characterized for the first time. Chemical derivatization of the natural products is not a new concept, but has not been previously applied on di-Rha substrates. Indeed, based on our results, it should be further explored as a platform for obtaining new diverse rhamnolipids with improved properties. It is worth mentioning that similar semi-synthetic approach was previously applied using natural sophorolipid mixture (another class of biological glycolipids) as a substrate in reaction with the sodium alkoxides to form the corresponding sophorolipid alkyl (methyl, ethyl, propyl, and butyl) esters derivatives (Zhang et al., 2004).
A series of 14 synthetic rhamnolipids, inspired by the natural Rha-C14-C14, naturally produced by Burkholderia plantarii, has been achieved using hydrophobically assisted switching phase synthesis and their physico-chemical properties, as well as bioactivity in terms of cytokine induction, were examined (Howe et al., 2006). Derivatives differed in acylation pattern, number of monosaccharide residues, and the charge resulting in a large variety of activities, from the complete lost to antagonistic activity. Indeed, generating three aromatic amide derivatives from two different sources of di-Rha, we have observed a general increase in antimicrobial, anti-biofilm formation, as well as biofilm disruption and antiproliferative activities. Carboxylic group of the bioactive compounds is often modified into various amide derivatives in order to mask free carboxylic 

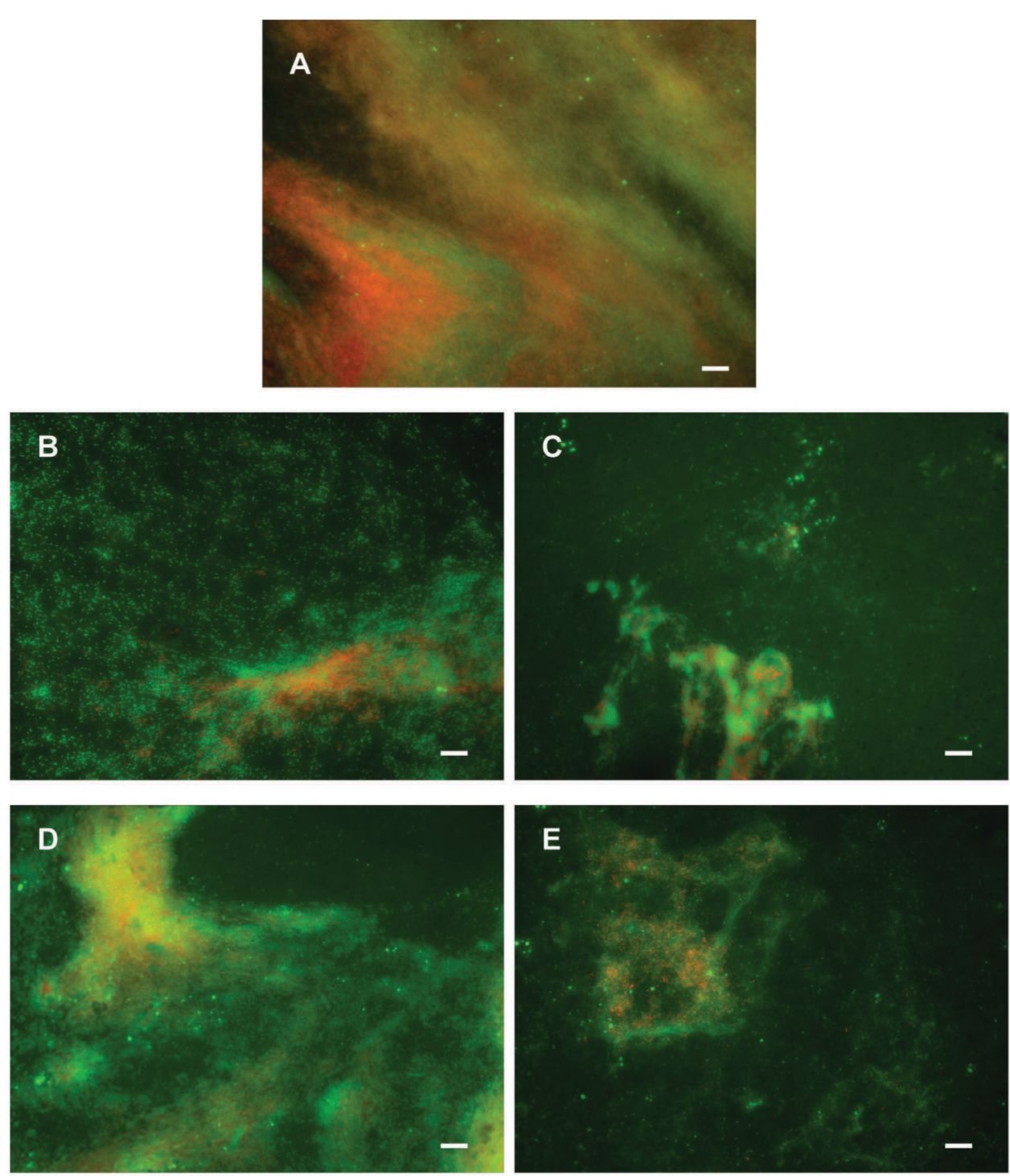

FIGURE 8 | Dispersion of $P$. aeruginosa PAO1 biofilms pre-formed on plastic surfaces (A) with $50 \mu \mathrm{g} \mathrm{mL}^{-1} \mathrm{~F} 3$ (B), di-Rha-Mor derivative from Lysinibacillus sp. BV152.1 (C), di-Rha (D), or di-Rha-Mor derivative from P. aeruginosa (E). Biofilms were stained with Syto9 (green) and PI (red), scale bars represent $10 \mu \mathrm{m}$.

group, commonly cause an increase in lipophilicity and tailor their biological activity (Chhikara et al., 2011; Guan et al., 2014). This was especially true for the di-Rha-Mor, that was determined to be the most potent derivative, and the only one that showed the antibacterial effect against both strains of $S$. aureus with MIC concentration values of $62.5 \mu \mathrm{g} \mathrm{mL}^{-1}$ (Table 1). Similarly, the introduction of morpholine moiety resulted in the increase of activity of 4-oxo- $4 \mathrm{H}$-pyrido[1,2-a]pyrimidine in its ability to potentiate the activity of Levofloxacin and Aztreonam against P. aeruginosa (Yoshida et al., 2006). Generally, morpholine derivatives are gaining considerable importance due to diverse biological activities including antibacterial and antiproliferative (Jakubowska et al., 2008; Hirokawa et al., 2009; Seelolla et al., 2014). Relatively small differences in the structure of the three amide derivatives generated in this study caused different biological effect. On the other side, the difference in the activities between rhamnolipid compounds from two sources was small and could be attributed to the fact that derivatives from the Lysinibacillus sp. BV152.1 contained the mixture of congeners of various carbon chain fatty acids, while the one from P. aeruginosa was pure C10-C10. Interestingly, derivative di-Rha-TBDS bearing bulky protective groups on the rhamnoses lost all the biological activities, suggesting that sugar moieties play a crucial role in the activity of these molecules. Indeed, this derivative had the lowest HLB and the highest $\log \mathrm{P}$ amongst studied molecules suggesting the loss of amphiphilic nature of rhamnolipid molecules.

Rhamnolipids are usually considered as non-toxic, however, several reports confirmed that in concentrations of 100 and 
TABLE 2 | Biofilm formation (\%) in the presence of rhamnolipids mixture (R90), di-rhamnolipids, and di-rhamnolipid derivatives from Lysinibacillus sp. BV152.1 and P. aeruginosa.

\begin{tabular}{|c|c|c|c|c|c|}
\hline Rhamnolipids $50 \mu \mathrm{g} \mathrm{mL}^{-1}$ & P. aeruginosa PAO1 & P. aeruginosa DM50 & S. aureus ATCC 25923 & S. aureus MRSA & S. marcescens ATCC 27117 \\
\hline \multicolumn{6}{|l|}{ Lysinibacillus sp. BV152.1 } \\
\hline di-Rha mixture (F3) & $50 \pm 5$ & $45 \pm 3$ & $78 \pm 12$ & $80 \pm 3$ & $38 \pm 13$ \\
\hline Rha-Bn & $60 \pm 2$ & $42 \pm 2$ & $4 \pm 0.5$ & $66 \pm 7$ & $20 \pm 4$ \\
\hline Rha-Pip & $50 \pm 2$ & $48 \pm 4$ & $4 \pm 0.5$ & $67 \pm 5$ & $20 \pm 4$ \\
\hline Rha-Mor & $20 \pm 3$ & $35 \pm 8$ & $11 \pm 2$ & $28 \pm 2$ & $12 \pm 1$ \\
\hline Rha-TBDMS & $115 \pm 10$ & $100 \pm 8$ & $88 \pm 12$ & $106 \pm 4$ & $85 \pm 1$ \\
\hline \multicolumn{6}{|l|}{$P$. aeruginosa } \\
\hline $\mathrm{R} 90$ & $54 \pm 5$ & $76 \pm 8$ & $43 \pm 6$ & $42 \pm 3$ & $15 \pm 2$ \\
\hline di-Rha & $50 \pm 3$ & $109 \pm 10$ & $37 \pm 4$ & $44 \pm 11$ & $20 \pm 5$ \\
\hline di-Rha-Bn & $36 \pm 3$ & $168 \pm 3$ & $39 \pm 2$ & $48 \pm 10$ & $50 \pm 7$ \\
\hline di-Rha-Pip & $39 \pm 4$ & $94 \pm 13$ & $31 \pm 2$ & $34 \pm 3$ & $20 \pm 3$ \\
\hline di-Rha-Mor & $50 \pm 4$ & $76 \pm 7$ & $27 \pm 2$ & $30 \pm 3$ & $14 \pm 1$ \\
\hline di-Rha-TBDMS & $95 \pm 7$ & $73 \pm 6$ & $88 \pm 10$ & $69 \pm 11$ & $117 \pm 4$ \\
\hline
\end{tabular}

Values are presented as mean $\pm S D$.

TABLE 3 | Biofilm biomass (\%) remained after biofilm dispersion with di-rhamnolipids and di-rhamnolipid derivatives from Lysinibacillus sp. BV152.1 and P. aeruginosa.

\begin{tabular}{|c|c|c|c|c|c|}
\hline Rhamnolipids $50 \mu \mathrm{g} \mathrm{mL}^{-1}$ & P. aeruginosa PAO1 & P. aeruginosa DM50 & S. aureus ATCC 25923 & S. aureus MRSA & S. marcescens ATCC 27117 \\
\hline \multicolumn{6}{|l|}{ Lysinibacillus sp. BV152.1 } \\
\hline di-Rha mixture (F3) & $53 \pm 5$ & $108 \pm 7$ & $115 \pm 13$ & $88 \pm 7$ & $243 \pm 3$ \\
\hline Rha-Bn & $46 \pm 2$ & $74 \pm 4$ & $64 \pm 5$ & $62 \pm 11$ & $37 \pm 2$ \\
\hline Rha-Pip & $52 \pm 5$ & $67 \pm 4$ & $87 \pm 5$ & $86 \pm 10$ & $180 \pm 7$ \\
\hline Rha-Mor & $32 \pm 2$ & $75 \pm 8$ & $68 \pm 10$ & $63 \pm 12$ & $42 \pm 4$ \\
\hline Rha-TBDMS & $80 \pm 6$ & $65 \pm 1$ & $110 \pm 22$ & $101 \pm 14$ & $99 \pm 8$ \\
\hline \multicolumn{6}{|l|}{ P. aeruginosa } \\
\hline di-Rha & $77 \pm 3$ & $135 \pm 10$ & $80 \pm 9$ & $82 \pm 7$ & $193 \pm 8$ \\
\hline di-Rha-Bn & $84 \pm 8$ & $70 \pm 6$ & $62 \pm 5$ & $55 \pm 10$ & $239 \pm 15$ \\
\hline di-Rha-Pip & $63 \pm 4$ & $59 \pm 5$ & $80 \pm 7$ & $54 \pm 2$ & $175 \pm 18$ \\
\hline di-Rha-Mor & $44 \pm 6$ & $67 \pm 8$ & $44 \pm 4$ & $70 \pm 6$ & $160 \pm 8$ \\
\hline di-Rha-TBDMS & $76 \pm 10$ & $78 \pm 10$ & $82 \pm 3$ & $120 \pm 10$ & $155 \pm 3$ \\
\hline
\end{tabular}

Values are presented as mean $\pm S D$.

$150 \mu \mathrm{g} \mathrm{mL}^{-1}$ they reveal significant toxicity and their antitumor activity has been focused on (Christova et al., 2013; Jiang et al., 2014). Rhamnolipids were reported to show considerable cytotoxicity on HeLa cells at a low concentration of $5 \mathrm{mg}$ $\mathrm{L}^{-1}$ (Lotfabad et al., 2010). However, we found that diRha were not toxic to normal human fibroblasts up to $100 \mu \mathrm{g} \mathrm{mL}^{-1}$, while the antiproliferative properties increased when cells were treated with amide derivatives. This is in line with the recent study confirming that rhamnolipids, like chemical surfactants, exhibited cytotoxicity by reducing the surface tension of culture medium rather than by changing its specific molecular structure, which had no selection on tumor cells and that natural rhamnolipids are not promising antitumor agents (Jiang et al., 2014), however, simple chemical alterations may increase their cytotoxicity. We also confirm findings that natural rhamnolipids have no direct antibacterial activity in concentrations of up to $500 \mu \mathrm{g} \mathrm{mL} \mathrm{m}^{-1}$ against $P$. aeruginosa, S. aureus, and S. marcescens, while others have reported MIC values in a range $4-32 \mathrm{mg} \mathrm{L}^{-1}$ against bacteria Enterobacter aerogenes, Proteus mirabilis, P. aeruginosa,
Salmonella Typhimurium, S. aureus, B. cereus, B. subtilis, Streptococcus faecalis and numerous fungal strains (Benincasa et al., 2004; Vatsa et al., 2010). Nevertheless, the most prominent activity of rhamnolipids is within their antibiofilm properties that have been confirmed in numerous studies, involving wide range of rhamnolipid producers and pathogenic biofilm forming strains (Irie et al., 2005; Dusane et al., 2011, 2012; De Rienzo and Martin, 2016; Díaz De Rienzo et al., 2016). Similar to activities observed in this work, purified di-rhamnolipids from P. aeruginosa ATCC 9027 completely disrupted $P$. aeruginosa PAO1 pre-formed biofilms

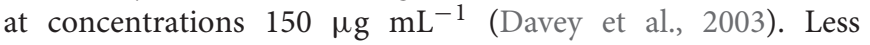
efficient were $P$. aeruginosa rhamnolipids against $B$. pumilus biofilms with BDIC $_{50}=580 \mu \mathrm{g} \mathrm{mL}^{-1}$ and complete biofilm inhibition at $50 \mathrm{mg} \mathrm{mL}^{-1}$ (Dusane et al., 2010). Generally lower in comparison to activities reported in this study but still potent biofilm disruption activities against important oral pathogens $\left[S\right.$. oralis $\left(\mathrm{BDIC}_{70}=750 \mu \mathrm{g} \mathrm{mL} \mathrm{m}^{-1}\right)$, Actinomyces naeslundii, Neisseria mucosa, and S. sanguinis (BDIC ${ }_{90}=190 \mu \mathrm{g}$ $\left.\mathrm{mL}^{-1}\right)$ ] and biofouling strains $S$. capitis and B. licheniformis 
$\left(\right.$ BDIC $_{50}=40 \mu \mathrm{g} \mathrm{mL} \mathrm{m}^{-1}$ ) were reported for rhamnolipids mixture purified from $B$. thailandensis containing mainly long chain Rha-Rha-C14-C14 di-rhamnolipids (Chebbi et al., 2017; Elshikh et al., 2017). Biofilm-related infections have been encountered in chronic diseases such as cystic fibrosis, otitis media, ventilator-associated pneumonia, and periodontitis, or in chronic wounds that have an impaired blood supply. Biofilms are often implicated in low sensitivity or resistance to antimicrobials. Thus, considering their broad-spectrum activity against bacteria and low cytotoxicity to human cells, di-Rha could be used to improve the effectiveness of antibiotics through biofilm inhibition, particularly for the treatment of chronic wound infections or in prophylactics of periodontal diseases. Besides, biofilms can be formed on the surface of medical devices (Bryers, 2008). Di-Rha and their derivatives could be used to functionalize the materials for medical usage in order to inhibit bacterial adhesion and formation of the biofilms on their surfaces, thus minimizing the spreading of the bacteria to the patients.

Recently, a series of synthetic rhamnolipid analogs including disaccharide maltose or cellobiose tethered with different aliphatic chains were prepared and their effect on the biofilm formation in $P$. aeruginosa rhamnolipid non-producing strain has been assessed (Zheng et al., 2017). As in our study, it has been shown that small structural details of these molecules are important for the bioactivities. Synthetic structural analogs of rhamnolipids promoted biofilm formation by non-rhamnolipid producing mutant at low concentrations but inhibited the biofilm formation at high concentrations such as 170 and $220 \mu \mathrm{g} \mathrm{mL}^{-1}$ (Zheng et al., 2017). Promotion of biofilm formation has not been observed during our study, while efficient inhibition of biofilm formation was observed at 10 and $50 \mu \mathrm{g} \mathrm{mL}^{-1}$.

\section{CONCLUSION}

Anti-biofilm activity guided screening of culture extracts lead to the identification of rhizosphere isolate Lysinibacillus sp.

\section{REFERENCES}

Abdel-Mawgoud, A. M., Lepine, F., and Deziel, E. (2010). Rhamnolipids: diversity of structures, microbial origins and roles. Appl. Microbiol. Biotechnol. 86, 1323-1336. doi: 10.1007/s00253-010-2498-2

Benincasa, M., Abalos, A., Oliveira, I., and Manresa, A. (2004). Chemical structure, surface properties and biological activities of the biosurfactant produced by Pseudomonas aeruginosa LBI from soapstock. Antonie Van Leeuwenhoek 85, 1-8. doi: 10.1023/B:ANTO.0000020148.45523.41

Berlanga, M., and Guerrero, R. (2016). Living together in biofilms: the microbial cell factory and its biotechnological implications. Microb. Cell Fact. 15:165. doi: 10.1186/s12934-016-0569-5

Bryers, J. D. (2008). Medical biofilms. Biotechnol. Bioeng. 100, 1-18. doi: 10.1002/ bit. 21838

Caiazza, N. C., Shanks, R. M., and O’Toole, G. A. (2005). Rhamnolipids modulate swarming motility patterns of Pseudomonas aeruginosa. J. Bacteriol. 187, 7351-7361. doi: 10.1128/JB.187.21.7351-7361.2005

Chebbi, A., Elshikh, M., Haque, F., Ahmed, S., Dobbin, S., Marchant, R., et al. (2017). Rhamnolipids from Pseudomonas aeruginosa strain W10; as antibiofilm/antibiofouling products for metal protection. J. Basic Microbiol. 57, 364-375. doi: 10.1002/jobm.201600658
BV152.1 producing a mixture of di-rhamnolipids. The dirhamnolipid fraction was found to be a potent anti-biofilm agent for the pathogenic $P$. aeruginosa PAO1 and DM50, S. aureus (ATCC 25923 and MRSA) and S. marcescens ATCC 27117 strains, while having no effect on the bacterial growth and showing low in vitro cytotoxicity against human fibroblasts. Isolated di-Rha from Lysinibacillus sp. BV152.1 and from the commercially available rhamnolipid mixture from $P$. aeruginosa were used as substrates for generation of semisynthetic amide derivatives, for the first time. Introducing amide functional group resulted in a general increase of biological activities. Thus, the semi-synthetic approach could be further explored for obtaining diverse rhamnolipids with improved activities.

\section{AUTHOR CONTRIBUTIONS}

All authors listed have made a substantial, direct and intellectual contribution to the work, and approved it for publication. LS and JN-R designed overall research and wrote the paper.

\section{FUNDING}

This study has been funded by a Research Grant 2015 by the European Society of Clinical Microbiology and Infectious Diseases (ESCMID) to LS and by the Ministry of Education, Science and Technological Development, Republic of Serbia (Grant Nos. 173048 and 172009).

\section{SUPPLEMENTARY MATERIAL}

The Supplementary Material for this article can be found online at: https://www.frontiersin.org/articles/10.3389/fmicb. 2017.02454/full\#supplementary-material

Chhikara, B. S., St. Jean, N., Mandal, D., Kumar, A., and Parang, K. (2011). Fatty acyl amide derivatives of doxorubicin: synthesis and in vitro anticancer activities. Eur. J. Med. Chem. 46, 2037-2042. doi: 10.1016/j.ejmech.2011.02.056

Christova, N., Tuleva, B., Kril, A., Georgieva, M., Konstantinov, S., Terziyski, I., et al. (2013). Chemical structure and in vitro antitumor activity of rhamnolipids from Pseudomonas aeruginosa BN10. Appl. Biochem. Biotechnol. 170, 676-689. doi: 10.1007/s12010-013-0225-z

Chrzanowski, L., Lawniczak, L., and Czaczyk, K. (2012). Why do microorganisms produce rhamnolipids? World J. Microbiol. Biotechnol. 28, 401-419. doi: 10.1007/s11274-011-0854-8

Compant, S., Duffy, B., Nowak, J., Clement, C., and Barka, E. A. (2005). Use of plant growth-promoting bacteria for biocontrol of plant diseases: principles, mechanisms of action, and future prospects. Appl. Environ. Microbiol. 71, 4951-4959. doi: 10.1128/AEM.71.9.4951-4959.2005

Costerton, J. W., Stewart, P. S., and Greenberg, E. P. (1999). Bacterial biofilms: a common cause of persistent infections. Science 284, 1318-1322. doi: 10.1126/ science.284.5418.1318

Coughlan, L. M., Cotter, P. D., Hill, C., and Alvarez-Ordonez, A. (2016). New weapons to fight old enemies: novel strategies for the (bio)control of bacterial biofilms in the food industry. Front. Microbiol. 7:1641. doi: 10.3389/fmicb.2016. 01641 
da Silva, D. P., Castaneda-Ojeda, M. P., Moretti, C., Buonaurio, R., Ramos, C., and Venturi, V. (2014). Bacterial multispecies studies and microbiome analysis of a plant disease. Microbiology 160, 556-566. doi: 10.1099/mic.0.074468-0

Davey, M. E., Caiazza, N. C., and O’Toole, G. A. (2003). Rhamnolipid surfactant production affects biofilm architecture in Pseudomonas aeruginosa PAO1. J. Bacteriol. 185, 1027-1036. doi: 10.1128/JB.185.3.1027-1036.2003

De Rienzo, M. A., and Martin, P. J. (2016). Effect of mono and di-rhamnolipids on biofilms pre-formed by Bacillus subtilis BBK006. Curr. Microbiol. 73, 183-189. doi: 10.1007/s00284-016-1046-4

Díaz De Rienzo, M. A., Stevenson, P. S., Marchant, R., and Banat, I. M. (2016). Pseudomonas aeruginosa biofilm disruption using microbial surfactants. J. Appl. Microbiol. 120, 868-876. doi: 10.1111/jam.13049

Djokic, L., Narancic, T., Nikodinovic-Runic, J., Savic, M., and Vasiljevic, B. (2011). Isolation and characterization of four novel Gram-positive bacteria associated with the rhizosphere of two endemorelict plants capable of degrading a broad range of aromatic substrates. Appl. Microbiol. Biotechnol. 91, 1227-1238. doi: 10.1007/s00253-011-3426-9

Dobler, L., Vilela, L. F., Almeida, R. V., and Neves, B. C. (2016). Rhamnolipids in perspective: gene regulatory pathways, metabolic engineering, production and technological forecasting. N. Biotechnol. 33, 123-135. doi: 10.1016/j.nbt.2015. 09.005

Dusane, D. H., Dam, S., Nancharaiah, Y. V., Kumar, A. R., Venugopalan, V. P., and Zinjarde, S. S. (2012). Disruption of Yarrowia lipolytica biofilms by rhamnolipid biosurfactant. Aquat. Biosyst. 8:17. doi: 10.1186/2046-9063-8-17

Dusane, D. H., Nancharaiah, Y. V., Zinjarde, S. S., and Venugopalan, V. P. (2010). Rhamnolipid mediated disruption of marine Bacillus pumilus biofilms. Colloids Surf. B Biointerfaces 81, 242-248. doi: 10.1016/j.colsurfb.2010.07.013

Dusane, D. H., Pawar, V. S., Nancharaiah, Y. V., Venugopalan, V. P., Kumar, A. R., and Zinjarde, S. S. (2011). Anti-biofilm potential of a glycolipid surfactant produced by a tropical marine strain of Serratia marcescens. Biofouling 27, 645-654. doi: 10.1080/08927014.2011.594883

Elshikh, M., Funston, S., Chebbi, A., Ahmed, S., Marchant, R., and Banat, I. M. (2017). Rhamnolipids from non-pathogenic Burkholderia thailandensis E264: physicochemical characterization, antimicrobial and antibiofilm efficacy against oral hygiene related pathogens. N. Biotechnol. 36, 26-36. doi: 10.1016/j.nbt. 2016.12.009

Fredsgaard, C., Moore, D. B., Chen, F., Clark, B. C., and Schneegurt, M. A. (2017). Prevalence of sucretolerant bacteria in common soils and their isolation and characterization. Antonie Van Leeuwenhoek 110, 995-1005. doi: 10.1007/ s10482-017-0873-z

Glisic, B. D., Senerovic, L., Comba, P., Wadepohl, H., Veselinovic, A., Milivojevic, D. R., et al. (2016). Silver(I) complexes with phthalazine and quinazoline as effective agents against pathogenic Pseudomonas aeruginosa strains. J. Inorg. Biochem. 155, 115-128. doi: 10.1016/j.jinorgbio.2015.11.026

Gong, Z., Peng, Y., and Wang, Q. (2015). Rhamnolipid production, characterization and fermentation scale-up by Pseudomonas aeruginosa with plant oils. Biotechnol. Lett. 37, 2033-2038. doi: 10.1007/s10529-015-1885-2

Govindasamy, V., Raina, S. K., George, P., Kumar, M., Rane, J., Minhas, P. S., et al. (2017). Functional and phylogenetic diversity of cultivable rhizobacterial endophytes of sorghum [Sorghum bicolor (L.) Moench]. Antonie Van Leeuwenhoek 110, 925-943. doi: 10.1007/s10482-017-0864-0

Guan, A., Liu, C., Yang, X., and Dekeyser, M. (2014). Application of the intermediate derivatization approach in agrochemical discovery. Chem. Rev. 114, 7079-7107. doi: 10.1021/cr4005605

Hirokawa, Y., Kinoshita, H., Tanaka, T., Nakamura, T., Fujimoto, K., Kashimoto, S., et al. (2009). Pleuromutilin derivatives having a purine ring. Part 2: influence of the central spacer on the antibacterial activity against Gram-positive pathogens. Bioorg. Med. Chem. Lett. 19, 170-174. doi: $10.1016 /$ j.bmcl.2008.10.123

Holscher, T., and Kovacs, A. T. (2017). Sliding on the surface: bacterial spreading without an active motor. Environ. Microbiol. 19, 2537-2545. doi: 10.1111/14622920.13741

Howe, J., Bauer, J., Andrä, J., Schromm, A. B., Ernst, M., Rössle, M., et al. (2006). Biophysical characterization of synthetic rhamnolipids. FEBS J. 273, 5101-5112. doi: 10.1111/j.1742-4658.2006.05507.x

Irie, Y., O’Toole, G. A., and Yuk, M. H. (2005). Pseudomonas aeruginosa rhamnolipids disperse Bordetella bronchiseptica biofilms. FEMS Microbiol. Lett. 250, 237-243. doi: 10.1016/j.femsle.2005.07.012
Jakubowska, J., Wasowska-Lukawska, M., and Czyz, M. (2008). STI571 and morpholine derivative of doxorubicin collaborate in inhibition of K562 cell proliferation by inducing differentiation and mitochondrial pathway of apoptosis. Eur. J. Pharmacol. 596, 41-49. doi: 10.1016/j.ejphar.2008. 08.021

Jiang, L., Shen, C., Long, X., Zhang, G., and Meng, Q. (2014). Rhamnolipids elicit the same cytotoxic sensitivity between cancer cell and normal cell by reducing surface tension of culture medium. Appl. Microbiol. Biotechnol. 98, 10187-10196. doi: 10.1007/s00253-014-6065-0

Kieser, T., Bibb, M. J., Buttner, M. J., Chater, K. F., and Hopwood, D. A. (2000). Practical Streptomyces Genetics. Norwich: John Innes Foundation.

Kim, L. H., Jung, Y., Kim, S.-J., Kim, C.-M., Yu, H.-W., Park, H.-D., et al. (2015). Use of rhamnolipid biosurfactant for membrane biofouling prevention and cleaning. Biofouling 31, 211-220. doi: 10.1080/08927014.2015.1022724

Li, Q. (2017). Rhamnolipid synthesis and production with diverse resources. Front. Chem. Sci. Eng. 11, 27-36. doi: 10.1007/s11705-016-1607-x

Lotfabad, T. B., Abassi, H., Ahmadkhaniha, R., Roostaazad, R., Masoomi, F., Zahiri, H. S., et al. (2010). Structural characterization of a rhamnolipid-type biosurfactant produced by Pseudomonas aeruginosa MR01: enhancement of dirhamnolipid proportion using gamma irradiation. Colloids Surf. B Biointerfaces 81, 397-405. doi: 10.1016/j.colsurfb.2010.06.026

Maier, R. M., and Soberón-Chávez, G. (2000). Pseudomonas aeruginosa rhamnolipids: biosynthesis and potential applications. Appl. Microbiol. Biotechnol. 54, 625-633. doi: 10.1007/s002530000443

Merritt, J. H., Kadouri, D. E., and O’Toole, G. A. (2005). Growing and analyzing static biofilms. Curr. Protoc. Microbiol. 22, 1B.1.1-1B.1.17. doi: 10.1002/ 9780471729259.mc01b01s00

Mihajlovic, L. E., Savic, A., Poljarevic, J., Vuckovic, I., Mojic, M., Bulatovic, M., et al. (2012). Novel methylene modified cyclohexyl ethylenediamine-N,N'-diacetate ligands and their platinum(IV) complexes. Influence on biological activity. J. Inorg. Biochem. 109, 40-48. doi: 10.1016/j.jinorgbio.2012.01.012

Muller, M. M., Hormann, B., Kugel, M., Syldatk, C., and Hausmann, R. (2011). Evaluation of rhamnolipid production capacity of Pseudomonas aeruginosa PAO1 in comparison to the rhamnolipid over-producer strains DSM 7108 and DSM 2874. Appl. Microbiol. Biotechnol. 89, 585-592. doi: 10.1007/s00253-0102901-Z

Muller, M. M., Hormann, B., Syldatk, C., and Hausmann, R. (2010). Pseudomonas aeruginosa $\mathrm{PAO} 1$ as a model for rhamnolipid production in bioreactor systems. Appl. Microbiol. Biotechnol. 87, 167-174. doi: 10.1007/s00253-010-2513-7

Muller, M. M., Kugler, J. H., Henkel, M., Gerlitzki, M., Hormann, B., Pohnlein, M., et al. (2012). Rhamnolipids-next generation surfactants? J. Biotechnol. 162, 366-380. doi: 10.1016/j.jbiotec.2012.05.022

Neu, T. R. (1996). Significance of bacterial surface-active compounds in interaction of bacteria with interfaces. Microbiol. Rev. 60, 151-166.

Nickzad, A., and Deziel, E. (2014). The involvement of rhamnolipids in microbial cell adhesion and biofilm development - an approach for control? Lett. Appl. Microbiol. 58, 447-453. doi: 10.1111/lam.12211

Ouoba, L. I. I., Vouidibio Mbozo, A. B., Thorsen, L., Anyogu, A., Nielsen, D. S., Kobawila, S. C., et al. (2015). Lysinibacillus louembei sp. nov., a spore-forming bacterium isolated from Ntoba Mbodi, alkaline fermented leaves of cassava from the Republic of the Congo. Int. J. Syst. Evol. Microbiol. 65, 4256-4262. doi: 10.1099/ijsem.0.000570

Pasquali, R. C., Taurozzi, M. P., and Bregni, C. (2008). Some considerations about the hydrophilic-lipophilic balance system. Int. J. Pharm. 356, 44-51. doi: 10.1016/j.ijpharm.2007.12.034

Piljac, A., Stipèević, T., Piljac-Žegarac, J., and Piljac, G. (2008). Successful treatment of chronic decubitus ulcer with $0.1 \%$ dirhamnolipid ointment. J. Cutan. Med. Surg. 12, 142-146. doi: 10.2310/7750.2008.07052

Pradhan, A. K., Pradhan, N., Sukla, L. B., Panda, P. K., and Mishra, B. K. (2014). Inhibition of pathogenic bacterial biofilm by biosurfactant produced by Lysinibacillus fusiformis S9. Bioprocess Biosyst. Eng. 37, 139-149. doi: 10.1007/ s00449-013-0976-5

Radivojevic, J., Skaro, S., Senerovic, L., Vasiljevic, B., Guzik, M., Kenny, S. T., et al. (2016). Polyhydroxyalkanoate-based 3-hydroxyoctanoic acid and its derivatives as a platform of bioactive compounds. Appl. Microbiol. Biotechnol. 100, 161-172. doi: 10.1007/s00253-015-6984-4

Rowson, C., and Townsend, R. (2016). Biofilms: prevention and treatment. Br. J. Hosp. Med. 77, 699-703. doi: 10.12968/hmed.2016.77.12.699 
Seelolla, G., Cheera, P., and Ponneri, V. (2014). Synthesis, antimicrobial and antioxidant activities of novel series of cinnamamide derivatives having morpholine moiety. Med. Chem. 4, 778-783. doi: 10.4172/2161-0444.100 0229

Sharma, A., Jansen, R., Nimtz, M., Johri, B. N., and Wray, V. (2007). Rhamnolipids from the rhizosphere bacterium Pseudomonas sp. GRP(3) that reduces damping-off disease in Chilli and tomato nurseries. J. Nat. Prod. 70, 941-947. doi: $10.1021 / \mathrm{np} 0700016$

Shetye, G. S., Singh, N., Jia, C., Nguyen, C. D., Wang, G., and Luk, Y. Y. (2014). Specific maltose derivatives modulate the swarming motility of nonswarming mutant and inhibit bacterial adhesion and biofilm formation by Pseudomonas aeruginosa. Chem. Biochem. 15, 1514-1523. doi: 10.1002/cbic.2014 02093

Singh, P. K., Schaefer, A. L., Parsek, M. R., Moninger, T. O., Welsh, M. J., and Greenberg, E. P. (2000). Quorum-sensing signals indicate that cystic fibrosis lungs are infected with bacterial biofilms. Nature 407, 762-764. doi: 10.1038/ 35037627

Sodagari, M., Wang, H., Newby, B. M., and Ju, L. K. (2013). Effect of rhamnolipids on initial attachment of bacteria on glass and octadecyltrichlorosilane-modified glass. Colloids Surf. B Biointerfaces 103, 121-128. doi: 10.1016/j.colsurfb.2012. 10.004

Stankovic, N., Radulovic, V., Petkovic, M., Vuckovic, I., Jadranin, M., Vasiljevic, B., et al. (2012). Streptomyces sp. JS520 produces exceptionally high quantities of undecylprodigiosin with antibacterial, antioxidative, and UV-protective properties. Appl. Microbiol. Biotechnol. 96, 1217-1231. doi: 10.1007/s00253012-4237-3

Stipcevic, T., Piljac, A., and Piljac, G. (2006). Enhanced healing of full-thickness burn wounds using di-rhamnolipid. Burns 32, 24-34. doi: 10.1016/j.burns.2005. 07.004

Varjani, S. J., and Upasani, V. N. (2017). Critical review on biosurfactant analysis, purification and characterization using rhamnolipid as a model biosurfactant. Bioresour. Technol. 232, 389-397. doi: 10.1016/j.biortech.2017.02.047

Vatsa, P., Sanchez, L., Clement, C., Baillieul, F., and Dorey, S. (2010). Rhamnolipid biosurfactants as new players in animal and plant defense against microbes. Int. J. Mol. Sci. 11, 5095-5108. doi: 10.3390/ijms11125095
Wittgens, A., Kovacic, F., Muller, M. M., Gerlitzki, M., Santiago-Schubel, B., Hofmann, D., et al. (2016). Novel insights into biosynthesis and uptake of rhamnolipids and their precursors. Appl. Microbiol. Biotechnol. 101, 2865-2878. doi: 10.1007/s00253-016-8041-3

Yan, W., Xiao, X., and Zhang, Y. (2017). Complete genome sequence of Lysinibacillus sphaericus LMG 22257, a strain with ureolytic activity inducing calcium carbonate precipitation. J. Biotechnol. 246, 33-35. doi: 10.1016/j.jbiotec. 2017.02.016

Yoshida, K.-I., Nakayama, K., Yokomizo, Y., Ohtsuka, M., Takemura, M., Hoshino, K., et al. (2006). MexAB-OprM specific efflux pump inhibitors in Pseudomonas aeruginosa. Part 6: exploration of aromatic substituents. Bioorg. Med. Chem. 14, 8506-8518. doi: 10.1016/j.bmc.2006.08.037

Zhang, L., Somasundaran, P., Singh, S. K., Felse, A. P., and Gross, R. (2004). Synthesis and interfacial properties of sophorolipid derivatives. Colloids Surf. A Physicochem. Eng. Asp. 240, 75-82. doi: 10.1016/j.colsurfa.2004.02.016

Zheng, H., Singh, N., Shetye, G. S., Jin, Y., Li, D., and Luk, Y.-Y. (2017). Synthetic analogs of rhamnolipids modulate structured biofilms formed by rhamnolipidnonproducing mutant of Pseudomonas aeruginosa. Bioorg. Med. Chem. 25, 1830-1838. doi: 10.1016/j.bmc.2017.01.042

Zhong, H., Jiang, Y., Zeng, G., Liu, Z., Liu, L., Liu, Y., et al. (2015). Effect of lowconcentration rhamnolipid on adsorption of Pseudomonas aeruginosa ATCC 9027 on hydrophilic and hydrophobic surfaces. J. Hazard. Mater. 285, 383-388. doi: 10.1016/j.jhazmat.2014.11.050

Conflict of Interest Statement: The authors declare that the research was conducted in the absence of any commercial or financial relationships that could be construed as a potential conflict of interest.

Copyright (c) 2017 Aleksic, Petkovic, Jovanovic, Milivojevic, Vasiljevic, NikodinovicRunic and Senerovic. This is an open-access article distributed under the terms of the Creative Commons Attribution License (CC BY). The use, distribution or reproduction in other forums is permitted, provided the original author(s) or licensor are credited and that the original publication in this journal is cited, in accordance with accepted academic practice. No use, distribution or reproduction is permitted which does not comply with these terms. 